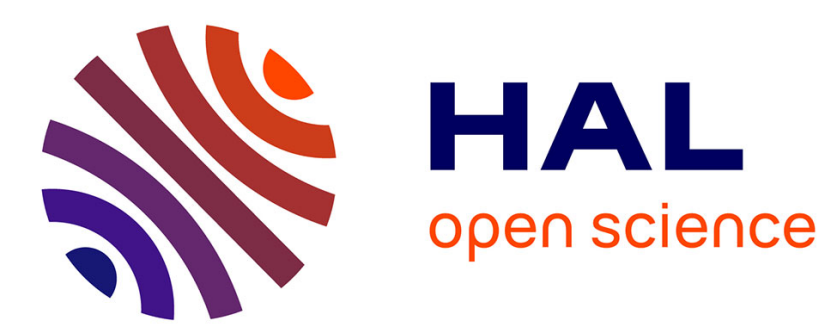

\title{
Multi-Rate Mass Transfer (MRMT) models for general diffusive porosity structures
}

Tristan Babey, Jean-Raynald de Dreuzy, Céline Casenave

\section{To cite this version:}

Tristan Babey, Jean-Raynald de Dreuzy, Céline Casenave. Multi-Rate Mass Transfer (MRMT) models for general diffusive porosity structures. Advances in Water Resources, 2015, 76, pp.146-156. 10.1016/j.advwatres.2014.12.006 . hal-01102049

\section{HAL Id: hal-01102049 \\ https://hal.inria.fr/hal-01102049}

Submitted on 12 Jan 2015

HAL is a multi-disciplinary open access archive for the deposit and dissemination of scientific research documents, whether they are published or not. The documents may come from teaching and research institutions in France or abroad, or from public or private research centers.
L'archive ouverte pluridisciplinaire HAL, est destinée au dépôt et à la diffusion de documents scientifiques de niveau recherche, publiés ou non, émanant des établissements d'enseignement et de recherche français ou étrangers, des laboratoires publics ou privés. 


\section{Multi-Rate Mass Transfer (MRMT) models for general \\ 2 diffusive porosity structures}

3 Tristan Babey ${ }^{1}$, Jean-Raynald de Dreuzy ${ }^{1}$, Céline Casenave ${ }^{2,3}$

$4{ }^{1}$ Géosciences Rennes (UMR CNRS 6118), Campus de Beaulieu, Université de Rennes 1, 535042 Rennes cedex, France

$6 \quad$ 2UMR INRA-SupAgro 0729 MISTEA, 2 place Viala 34060 Montpellier, France

$7 \quad{ }^{3}$ MODEMIC project-team, INRA/INRIA, Sophia-Antipolis, France

8 Corresponding author: Tristan Babey, +33646204318, tristan.babey@univ-rennes1.fr

9 Highlights:

- A mobile-immobile transport model with a structured immobile domain is proposed

- Structured INteracting Continua-SINC generalize Multiple INteracting ContinuaMINC

- Whatever the SINC structure, a unique equivalent MRMT model exists

- MRMT models with only very few rates accurately model conservative transport

- We propose a robust numerical identification of the first few rates

\section{ABSTRACT}

We determine the relevance of Multi-Rate Mass Tansfer (MRMT) models to general diffusive porosity structures. To this end, we introduce Structured INteracting Continua models (SINC) as the combination of a finite number of diffusion-dominated interconnected immobile zones exchanging with an advection-dominated mobile domain. It directly extends Multiple INteracting Continua framework [Pruess and Narasimhan, 1985] by introducing a structure in the immobile domain, coming for example from the dead-ends of fracture clusters or poorlyconnected dissolution patterns. We demonstrate that, whatever their structure, SINC models can be made equivalent in terms of concentration in the mobile zone to a unique Multi-Rate Mass Transfer (MRMT) model [Haggerty and Gorelick, 1995]. We develop effective shapefree numerical methods to identify its few dominant rates, that comply with any distribution of rates and porosities. We show that differences in terms of macrodispersion are not larger than $50 \%$ for approximate MRMT models with only one rate (double porosity models), and drop down to less than $0.1 \%$ for five rates MRMT models. Low-dimensional MRMT models 
30 accurately approach transport in structured diffusive porosities at intermediate and long times 31 and only miss early responses.

32

33 Keywords: Porous media; Solute transport; Mobile-immobile models; Multi-Rate Mass 34 Transfer; 


\section{Introduction}

Transport in complex geological environments results in part from the interactions between fast advective-dominated transport in a localized "mobile porosity" and slow diffusivedominated transport in extensive "immobile porosities". It is the case of the fracture-matrix systems [Neretnieks, 1980; Tang et al., 1981] and of the highly heterogeneous porous media [Fernandez-Garcia et al., 2009; Golfier et al., 2007; Gotovac et al., 2009; Willmann et al., 2008]. When diffusive times in the immobile zones become much larger than the characteristic advective time in the mobile zone, transport becomes anomalous with nonGaussian concentration plumes, more extensive spreading and mixing, slow transit times, and broad ranges of solute retardation times [Berkowitz et al., 2006; Dentz et al., 2004]. Such transport mechanisms and exchanges are at the root of numerous anomalous transport modeling frameworks [Benson and Meerschaert, 2009; Benson et al., 2000; Berkowitz and Scher, 1998; Carrera et al., 1998; Cushman and Ginn, 2000; Haggerty and Gorelick, 1995] and can be highly effective in the interpretative and predictive phases of laboratory and field experiments [Benson et al., 2001; Berkowitz et al., 2000; Gouze et al., 2008; Haggerty et al., 2001; Haggerty et al., 2004]. Anomalous transport ultimately stems from some extended distribution whether it is a waiting time distribution as in Continuous Time Random Walk (CTRW) or a rate-porosity distribution as in Mutli-Rate Mass Transfert (MRMT) [Dentz and Berkowitz, 2003; Neuman and Tartakovsky, 2009; Silva et al., 2009]. For MRMT models, while these distributions can take very different shapes [Haggerty et al., 2000], only some power-law distributions are effectively related to diffusive processes in 1D, 2D or 3D inclusions [Carrera et al., 1998; Haggerty and Gorelick, 1995] or to anomalous diffusive processes in fractal-like structures [Haggerty, 2001]. Diffusive structures may however be

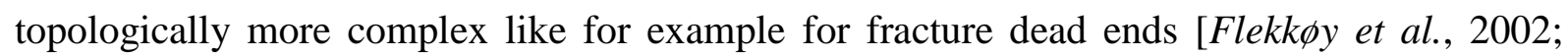
Sornette et al., 1993], fracture-matrix interactions [Jardine et al., 1999; Karimi-Fard et al., 2006; Sudicky and Frind, 1982; Tang et al., 1981; Tsang, 1995], or dissolution patterns in porous media [Golfier et al., 2002; Luquot et al., 2014] (Figure 1).

In this article, we show that the MRMT framework is general to all diffusive architectures that can be modeled as a finite number of interconnected continua (Figure 1). The notion of continuum comes from the double porosity and Multiple INteracting Continua (MINC) concepts introduced initially for fracture-matrix systems [Pruess and Narasimhan, 1985; Warren et al., 1963]. The double porosity model is the classical diffusive interaction of advective-diffusive processes in a mobile zone with a single immobile zone like in double- 
68

69

porosity models [Warren et al., 1963]. The Multiple INteracting Continua (MINC) framework models matrix diffusion as diffusive-like exchanges within a succession of "continua", identified to the elementary cells issued by a finite-difference discretization of the diffusion process in the matrix (Figure 2a) [Pruess, 1992; Pruess and Narasimhan, 1985]. The denomination of multiple continua is a direct generalization of the double porosity concept of Warren and Root [1963]. We propose to further generalize the notion of interacting continua to any immobile zones structure where diffusive-like exchanges intervene between any connected zones or continua. Because of the potential importance of structure on diffusion, we denote these models as Structured INteracting Continua (SINC). SINC models include a wide range of structures going from elementary branching and loops (Figure $2 b$ and $c$ ) to more involved dissolution patterns (Figure 2d). They would typically be derived from the coarse discretization of diffusion processes in dead-end porosity structures [Gouze et al., 2008; Noetinger and Estebenet, 2000]. We define SINC models in section 2, with their exact relation to the MRMT and MINC models. We show in section 3 that any SINC model is equivalent in terms of transport to a unique MRMT model of the same dimension, i.e. with the same number of immobile zones. We develop efficient numerical methods in section 4 to identify lower-dimension but highly accurate approximate MRMT models. 


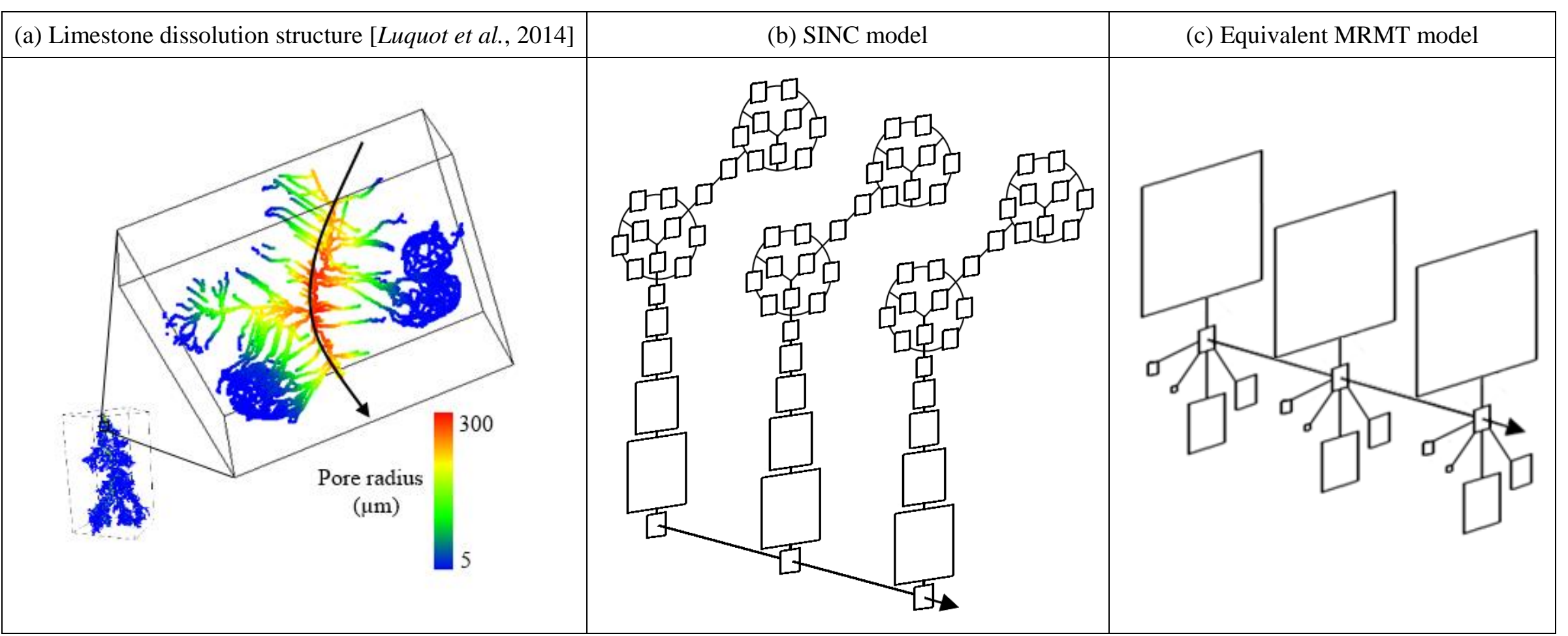

Figure 1: (a) Skeleton of a dissolution feature in an oolitic limestone, observed by X-ray micro-tomography [Luquot et al., 2014]. The dissolving acidic solution percolates from top to bottom on the general view (bottom left). Its pH increases from top to bottom and from inside out of the main flow path indicated by the curved arrow on the detailed view (top right). The acid dissolves preferentially the calcite cement surrounding the oolites, the size of the pores progressively decreases away from the main flow path, and the organization of the pores becomes more complex. zone materialized by the arrow. (c) Equivalent MRMT model with the 5 most important rates as determined by the numerical methods set up in section 4 . The size of the boxes scales with the porosity affected to the rates labeled by triangles in Figure 7. 


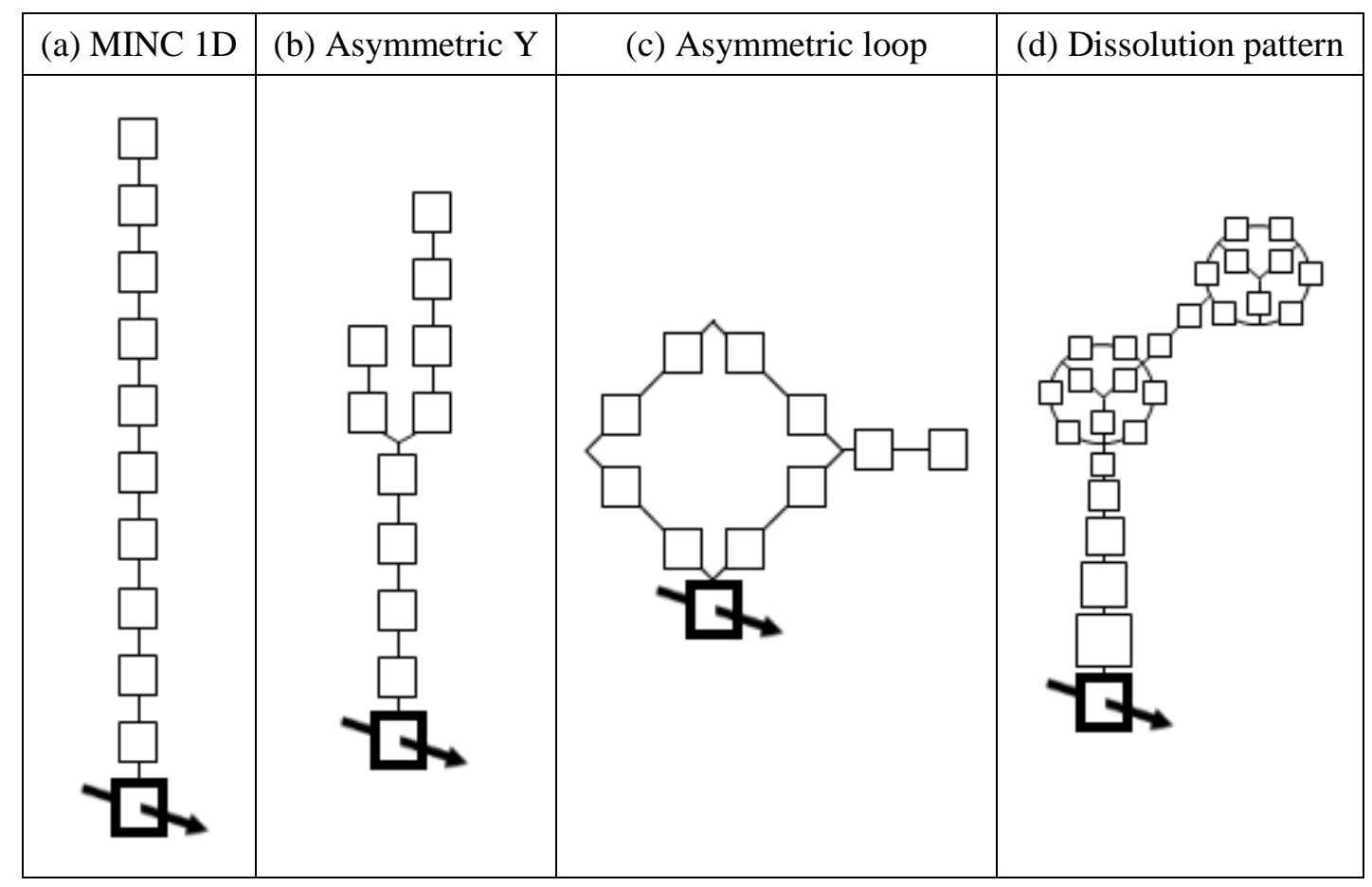

93 Figure 2: Examples of Structured INteracting Continua (SINC) used to illustrate and validate the numerical identification methods of the equivalent MRMT models. From left to right, the diffusive porosity structures are (a) the classical Multiple INteracting Continua (MINC) [Pruess and Narasimhan, 1985], (b) an asymmetric Y with a single junction, (c) an asymmetric loop, and (d) the dissolution structure presented in Figure 1. The size of the immobile cells is proportional to their porosity and the distance along the immobile structure is to scale. The mobile zone is represented by the thick black box with the crossing arrow. Its size has been exaggerated 10 times to be clearly marked. To be comparable, the four structures have the same total porous volume and the same radius of gyration taken with respect to the mobile zone. 
101 We present the Structured INteracting Continua framework (SINC) and show how it relates to existing models like Multi-Rate Mass Transfer (MRMT) and Multiple INteracting Continua

103 (MINC).

104 The SINC model is made up of a continuous 1-D mobile zone in interaction with a finite number of interconnected immobile zones (Figure 1). In the continuous mobile zone, solutes are transported by advection, dispersion and diffusion. Between the mobile zone and the immobile zones as well as between the immobile zones, concentration exchanges are

108 diffusive-like, i.e. directly proportional to the difference of concentrations. This model can be 109 generically expressed as:

$$
\frac{\partial U}{\partial t}-A U=L\left(R_{m} U\right)
$$

110 where $U=\left[\begin{array}{llll}c_{m}(x, t) & c_{i m}^{1}(x, t) & \cdots & c_{i m}^{N}(x, t)\end{array}\right]^{T}$ is the vector of dimension $N+1$ made up of the

111 concentrations in the mobile zone $c_{m}(x, t)$ and in the $N$ immobile zones $c_{i m}^{i}(x, t)$ with $112 i=1, \ldots, N . A$ is the $(N+1, N+1)$ interaction matrix characterizing the diffusive-like 113 concentration exchanges between the immobile zones and with the mobile zone. $R_{m}$ is the 114 restriction matrix to the mobile zone:

$$
R_{m}(i, j)=\delta(i-1) \delta(j-1)
$$

$115 L$ is the transport operator in the mobile zone:

$$
L\left(c_{m}\right)=-\frac{q}{\phi_{m}} \frac{\partial c_{m}}{\partial x}+d_{m} \frac{\partial^{2} c_{m}}{\partial x^{2}}
$$

116 with $q, \phi_{m}$ and $d_{m}$ the Darcy flow, porosity and dispersion coefficient in the mobile zone.

117 The physical properties of the mobile and immobile domains are homogeneous along the 118 mobile domain. The interaction matrix $A$ is equal to the matrix $M$ deriving from the diffusive119 like mass exchanges between the different zones, corrected by their porosities:

$$
A=-\Phi^{-1} M
$$



mobile zone $\phi_{\mathrm{m}}$ and to the $N$ immobile zones $\phi_{i m}^{i}$ with $i=1, \ldots, N$ :

$$
\left\{\begin{array}{l}
\Phi(1,1)=\phi_{m} \\
\Phi(i, j)=\phi_{i m}^{i-1} \delta(i, j) \text { for }(i, j) \neq(1,1)
\end{array} .\right.
$$

122

123

124

The matrix $M$ expresses rates of mass exchange. The dimension of its elements is therefore the inverse of a time. As exchanges are diffusive-like, $M$ is a $M$-matrix, i.e. $M$ is symmetric, its diagonal elements are positive, its off-diagonal elements are negative or equal to zero, and the sum of its elements along each of its rows is equal to zero. We underline that it is the matrix $M$ that is symmetric and generally not the interaction matrix $A$ that also integrates the differences in porosities. The interaction matrix $A$ registers the connectivity of the different zones through the position of its non-zero off-diagonal elements, the strength of the interactions is determined by porosity ratios and exchange rates. Figure 3 a shows the example of the interaction matrix for the asymmetric $\mathrm{Y}$ structure (cf Figure 2b). The branching architecture leads to a compact interaction matrix, which values are all on the three principal diagonals except at the branching node. The interaction matrix is scaled by the inverse of the mean diffusion time in the immobile structure $\tau$. We define $\tau$ as the quadratic mean distance of the immobile zones to the mobile zone divided by the diffusion coefficient between two zones.

The SINC framework generalizes Multiple INteracting Continua (MINC) [Pruess and Narasimhan, 1985]. MINC models are obtained by the finite-difference discretization of diffusion in a 1-D homogeneous medium. In such a case, the interaction matrix $A$ is simply tri-diagonal. The term "continuum" comes from the continuity along the mobile zone like in the dual-porosity concept [Warren et al., 1963]. It is consistent with our concept of a continuous formalism of transport along the mobile zone and a finite number of interacting continua in the immobile direction.

The SINC framework also generalizes Multiple-Rate Mass Transfer models with a finite number of rates [Carrera et al., 1998; Haggerty and Gorelick, 1995]. In MRMT models as defined by Haggerty et al. [1995], the immobile domain consists in a distribution of subdomains exchanging exclusively with the mobile domain. The star-like connectivity structure leads to an arrow-type broad-width interaction matrix (Figure 3b). Each sub-domain is characterized by its rate of transfer $\alpha_{i}$ and its porosity $\phi_{i m}^{i}$, with $\alpha_{i}<\alpha_{i+1}$ [Haggerty and 
149 Gorelick, 1995]. When defined with a finite number of rates, MRMT models can be recovered 150 by fixing

$$
\begin{aligned}
& \left\{\begin{array}{l}
M(i, j)=0 \quad \text { for } i>1, j>1 \text { and } i \neq j \\
M(i, 1)=M(1, i)=-\phi_{i m}^{i-1} \alpha_{i-1} \quad \text { for } i>1 \\
M(i, i)=-\sum_{j, j \neq i} M(i, j)
\end{array}\right. \\
& \left\{\begin{array}{l}
\Phi(1,1)=\phi_{m} \\
\Phi(i, j)=\phi_{i m}^{i-1} \delta(i, j) \quad \text { for }(i, j) \neq(1,1)
\end{array}\right.
\end{aligned}
$$

151 Because they are defined in algebraic terms, SINC models can only represent MRMT models 152 with finite number of rates. They are rigorously not equivalent to $1 \mathrm{D}, 2 \mathrm{D}$ and $3 \mathrm{D}$ diffusion 153 models that themselves correspond to an infinite number of exchange rates [Haggerty and 154 Gorelick, 1995]. However they offer very accurate approximations when taking only a finite 155 number of rates, especially as the porosities $\phi_{i m}^{i}$ decrease as a power law of $i$. SINC models 156 generalize MRMT models defined with a finite number of rates but remain different to 157 MRMT models defined by an infinite serie of rates or by a continuous rate-porosity function. 158 Our objective in this article is not to produce any kind of generalization of MRMT models but 159 rather to determine how complex diffusive porosity structures can be approached by simpler reduced models. 


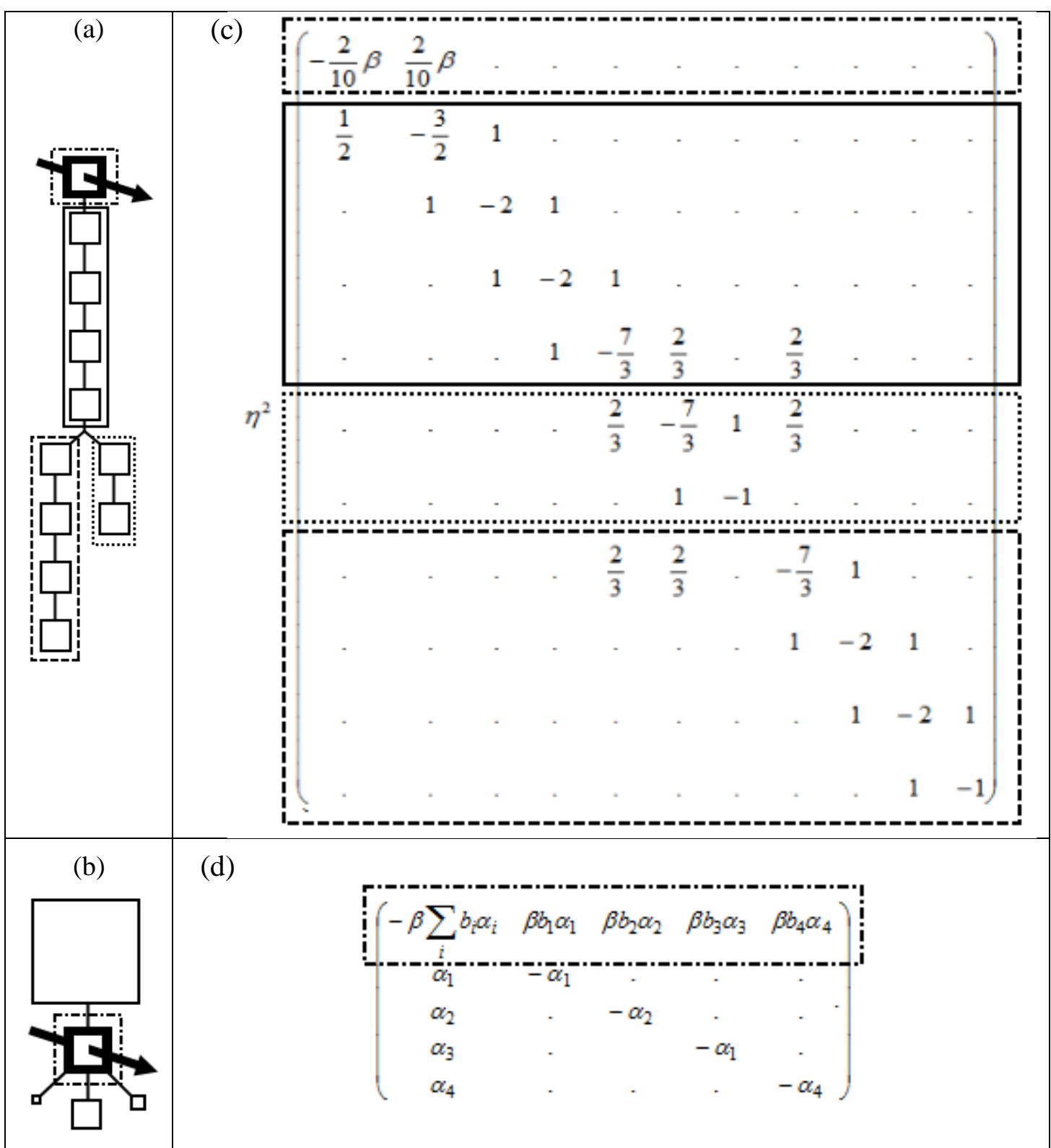

161 Figure 3: Diffusive porosity structures represented as cross-sections transversal to the mobile

162 zone direction ((a),(b)), with their associated interaction matrix $A((\mathrm{c}),(\mathrm{d}))$ for the asymmetric

$163 \mathrm{Y}$ (top) and MRMT structures (bottom). Dotted frames around subsets of the immobile

164 porosity structures ((a) and (b)) and around matrix lines ((c) and (d)) show how structures are

165 translated in matrix form. Parameters for the asymmetric Y structure are taken from Table 1

166 and the multiplicative factor $\eta(\eta=5.015)$ is equal to the ratio of the distance between two

167 consecutive immobile zones to the radius of gyration of the immobile domain to the mobile 168 zone. $\beta$ is the ratio of the total immobile porosity to the mobile porosity. 
170 Following up the work of Haggerty and Gorelick [1995], we define the equivalence relation 171 to a MRMT model as the identity of the mobile concentration at any time. To prove the 172 equivalence of the SINC model to a MRMT model, we first decompose the interaction matrix $173 A$ in two parts, consisting in the exchanges between the immobile zones only for the first part

174 and in the exchanges between the mobile zone and the immobile zones for the second one:

$$
A=\left(\begin{array}{cccc}
0 & \cdots & \cdots & 0 \\
\vdots & & & \\
\vdots & & \widehat{A} & \\
0 & & &
\end{array}\right)+\left(\begin{array}{cccc}
A_{11} & A_{12} & \cdots & A_{1, N+1} \\
A_{21} & & & \\
\vdots & & 0 & \\
A_{N+1,1} & & &
\end{array}\right)
$$

175 with $\widehat{A}=A(2 \ldots N+1,2 \ldots N+1)$. As shown in Appendix $A$, the matrix $\widehat{A}$ can be diagonalized

$$
\widehat{A}=\widehat{R} \Lambda \widehat{R}^{-1}
$$

176 with $\Lambda$ the diagonal matrix made up of the eigenvalues of $\hat{A}$, which are all real and negative, 177 and $\widehat{R}$ the matrix of the eigenvectors of $\widehat{A}$, defined each up to a multiplicative constant. To 178 fix the eigenvalues decomposition, as $\Lambda$ is defined up to a permutation of its diagonal 179 elements, we sort the eigenvalues according to their absolute value by increasing order $180\left|\Lambda_{i, i}\right| \leq\left|\Lambda_{i+1, i+1}\right|$. In the new coordinate system defined by the eigenvectors, there are no more 181 exchanges between the immobile zones. All exchanges are made directly between the 182 immobile zones and the mobile zone. To characterize the precise nature and extent of these 183 exchanges, we propagate the change of the coordinate system to the exchanges with the 184 mobile zone

$$
\left(\begin{array}{cccc}
A_{11} & A_{12} & \cdots & A_{1, N+1} \\
A_{21} & & & \\
\vdots & & 0 & \\
A_{N+1,1} & & &
\end{array}\right)=R\left(\begin{array}{cccc}
A_{11} & B_{12} & \cdots & B_{1, N+1} \\
B_{21} & & & \\
\vdots & & 0 & \\
B_{N+1,1} & & &
\end{array}\right) R^{-1}
$$

185 with the $(N+1, N+1)$ matrix $R$ and its inverse $R^{-1}$ defined as 


$$
R=\left(\begin{array}{cccc}
1 & 0 & \cdots & 0 \\
0 & & & \\
\vdots & & \widehat{R} & \\
0 & & &
\end{array}\right) \text { and } R^{-1}=\left(\begin{array}{cccc}
1 & 0 & \cdots & 0 \\
0 & & & \\
\vdots & & \widehat{R}^{-1} & \\
0 & &
\end{array}\right)
$$

186 In this transformation, the mobile zone remains unchanged consistently with our objective to 187 reorganize only the immobile zones without interfering with the concentration in the mobile 188 zone. The full transformation defined by $R$ is applied to the matrix $A$ following its 189 decomposition in equation (7)

$$
A=R\left(\begin{array}{cccc}
0 & \cdots & \cdots & 0 \\
\vdots & & & \\
\vdots & & \Lambda \\
0 & & &
\end{array}\right) R^{-1}+R\left(\begin{array}{cccc}
A_{11} & B_{12} & \cdots & B_{1, N+1} \\
B_{21} & & & \\
\vdots & & 0 & \\
B_{N+1,1} & & &
\end{array}\right) R^{-1}
$$

190 which can be finally expressed by a simple factorization as

$$
A=R B R^{-1} \text { with } B=\left(\begin{array}{cccc}
A_{11} & B_{12} & \cdots & B_{1, N+1} \\
B_{21} & & & \\
\vdots & & \Lambda & \\
B_{N+1,1} & &
\end{array}\right)
$$

191 Finally, as the restriction matrix to the mobile zone $R_{m}$ (equation (2)) commutes with $R^{-1}$, we 192 can write the full model as

$$
\frac{\partial R^{-1} U}{\partial t}-B R^{-1} U=L\left(R_{m} R^{-1} U\right)
$$

193 To be representative of a MRMT model, $B$ should be an arrow matrix, the sum of its elements 194 over each of its rows should be zero, and all its non-diagonal elements must be either positive 195 or equal to zero. In Appendix B, we show that this can be obtained by adjusting the norm of 196 the eigenvectors in $\hat{R}$. The characteristics of this MRMT model are then determined by a 197 simple identification of equation (6) with equation (12): 


$$
\left\{\begin{array}{l}
\alpha_{i}=-\Lambda_{i} \\
\phi_{i m}^{i}=\phi_{m} \frac{B_{1, i+1}}{B_{i+1,1}}=\phi_{m} \frac{B_{1, i+1}}{-\Lambda_{i}}
\end{array} .\right.
$$

198 While this algebraic identification method can already be widely used to determine equivalent

199 MRMT models, it faces some numerical limitations. The diagonalization process becomes 200 challenging when $\hat{A}$ becomes large, limiting the range of the identification to not too complex architectures and/or coarse discretizations of the immobile domain. The immobile porosity structure may be composed of a large number of cells, while a much smaller number of rates may be necessary to get highly accurate equivalent MRMT models. To address these limitations, we propose an alternative approximate numerical identification method.

\section{Approximate numerical identification method of the MRMT model equivalent to a}

\section{SINC model}

We first develop the numerical methodology and secondly apply it to the four examples of

208 Figure 2. Because of their widely differing structures, these four SINC examples are thought 209 to be a good basis for testing and illustrating the numerical methods. The first one is the classical MINC taken as reference. The two next ones were chosen for their elementary branching and looping connectivity patterns (Figure $2 b$ and $c$ ). Any more involved patterns

212 like the dissolution pattern of Figure $2 \mathrm{~d}$ will be some kind of combination of these elementary 213 structures. These four examples are comparable in the sense that they have the same mobile 214 properties, the same overall immobile to mobile porosity ratio $\beta$, and the same mean quadratic 215 diffusion time $\tau$ as defined in section 2 (Table 1). The dispersivity in the mobile zone $216 d_{m} \bar{I}\left(q / \phi_{m}\right)$ divided by the effective dispersivity due to the exchanges with the immobile zone 217 defined as $\tau\left(q / \phi_{m}\right)$ is taken equal to $5.10^{-5}$, i.e. much smaller than 1 , so that dispersive effects 218 come predominantly from the mobile/immobile exchanges. For the same reason, $\beta$ is taken 219 much larger than $1(\beta=100)$. 


\begin{tabular}{|c|c|}
\hline Parameter & Value \\
\hline$\beta$ & 100 \\
\hline$d_{m} /\left(\tau\left(q / \phi_{m}\right)^{2}\right)$ & $5.10^{-5}$ \\
\hline$\sigma_{0} /\left(q \tau / \phi_{m}\right)$ & $3.10^{-3}$ \\
\hline$x_{\max } / \sigma_{0}$ & 100 \\
\hline$d x / \sigma_{0}$ & 0.2 \\
\hline$d t / \tau$ & $5.10^{-4}$ \\
\hline
\end{tabular}

220 Table 1: Parameters used for the simulation of section 4.2 with the characteristic diffusion 221 time $\tau$ and the consecutive distance covered by advection in the mobile zone $q \tau / \phi_{m}$ as 222 temporal and spatial dimensional parameters. $\beta$ is the immobile to mobile porosity ratio. $223 d_{m} /\left(\tau\left(q / \phi_{m}\right)^{2}\right)$ is the dimensionless dispersion in the mobile zone. $\sigma_{0} /\left(q \tau / \phi_{m}\right)$ is the 224 dimensionless standard deviation of the initial Gaussian concentration profile. $x_{\max }$ is the 225 extension of the simulation domain in the direction of the mobile zone, $d x$ is the spatial step 226 along the mobile zone and $d t$ is the time step. 


\subsection{Methodology}

228 To numerically approximate the MRMT model equivalent to a SINC model, we consider the particular case of the discharge of the immobile zones to the mobile zone. The mass discharged to the mobile zone from an initially homogeneous immobile concentrations $c_{0}$ is fitted by a combination of exponential functions typical of the MRMT model. The residual mass per unitary volume $m(t)$ in the immobile domains is fitted by its MRMT counterpart $\gamma(t)$ given by:

$$
\gamma(t)=\sum_{i=1}^{N} c_{0} \phi_{i m}^{i} \exp \left(-\alpha_{i} t\right)
$$

234 where $\alpha_{i}$ and $\phi_{i m}^{i}, i=1 \ldots N$ are the $N$ rates and immobile porosities defined in equation (6).

235 In Appendix $C$, we develop an optimization method to identify the $\alpha$ and $\phi_{i m}$ series. We 236 further illustrate its application to the cases $N=1$ and $N=2$ in Appendix D.

237 The advantage of this method over the previous algebraic method is to be flexible in terms of 238 the number of rates $N$ to identify. It also prioritizes the identification of the rates and 239 immobile porosities having a significant impact on transport. In the following we determine 240 the number of rates that should be identified to model accurately macrodispersive processes.

\section{$241 \quad 4.2 \quad$ Simulations and results}

242 We analyze the influence of the number of rates $N$ on the reproduction of macrodispersion for 243 the four structures displayed in Figure 2 for $N$ ranging from 1 to 5. Identification is performed 244 according to the methodology presented before and with a logarithmic sampling of times 245 starting at the time for which the relative mass discharge to the mobile zone is lower than $10^{-3}$ and ending at the time for which the relative residual mass itself is lower than $10^{-4}$.

247 Simulations of transport for the SINC and approximate MRMT models are further performed 248 with identical Gaussian concentration profiles in the mobile and immobile domains

$$
c_{m}(x, t=0)=c_{i m}(x, t=0)=\frac{c_{\max }}{\sigma_{0} \sqrt{2 \pi}} \exp \left(-\frac{\left(x-x_{0}\right)^{2}}{2 \sigma_{0}^{2}}\right)
$$

249 where $x_{0}, \sigma_{0}$ and $c_{\max }$ are the mean, standard deviation and maximum concentration of the 250 Gaussian profile. $\sigma_{0}$ is taken small enough so that the initial plume size has minor effects on 251 the overall dispersion (Table 1). 
252 We compute for all models the effective dispersion coefficient $D$ as

$$
D=\frac{1}{2} \frac{d \sigma_{x}^{2}}{d t}
$$

253 with $\sigma_{\mathrm{x}}$ the plume spreading

$$
\sigma_{x}^{2}=m_{2} / m_{0}-\left(m_{1} / m_{0}\right)^{2}
$$

254 The spatial moments of concentration $m_{k}$ are given by

$$
m_{k}(t)=\int_{x=0}^{\infty} \sum_{i=1}^{N+1} x^{k} \Phi(i, i) U(x, t, i) d x
$$

255 We assess the quality of the MRMT model for modeling dispersion through the quadratic 256 mean of the relative difference in effective dispersion of the SINC and MRMT models:

$$
\operatorname{diff}\left(D_{\text {SINC }}, D_{M R M T}\right)=\sqrt{\int_{t}\left[\frac{D_{S I N C}(t)-D_{M R M T}(t)}{\left(D_{S I N C}(t)+D_{M R M T}(t)\right) / 2}\right]^{2} d t / \int_{t} d t} .
$$

257 This criterion is sensitive to differences in dispersion on a broad time range from the initial 258 time to the time at which dispersion becomes constant.

259 Classical numerical methods were used to solve both the exchanges within the immobile 260 zones and the advective-dispersive transport in the mobile zone [de Dreuzy et al., 2013]. They 261 were validated on a set of immobile structures rigorously equivalent to the MINC model 262 (Figure 4). In those cases, $\operatorname{diff}\left(D_{S I N C}, D_{M I N C}\right)$ was of the order of $10^{-11}$ and much smaller than 263 any differences recorded in the analysis performed hereafter. 


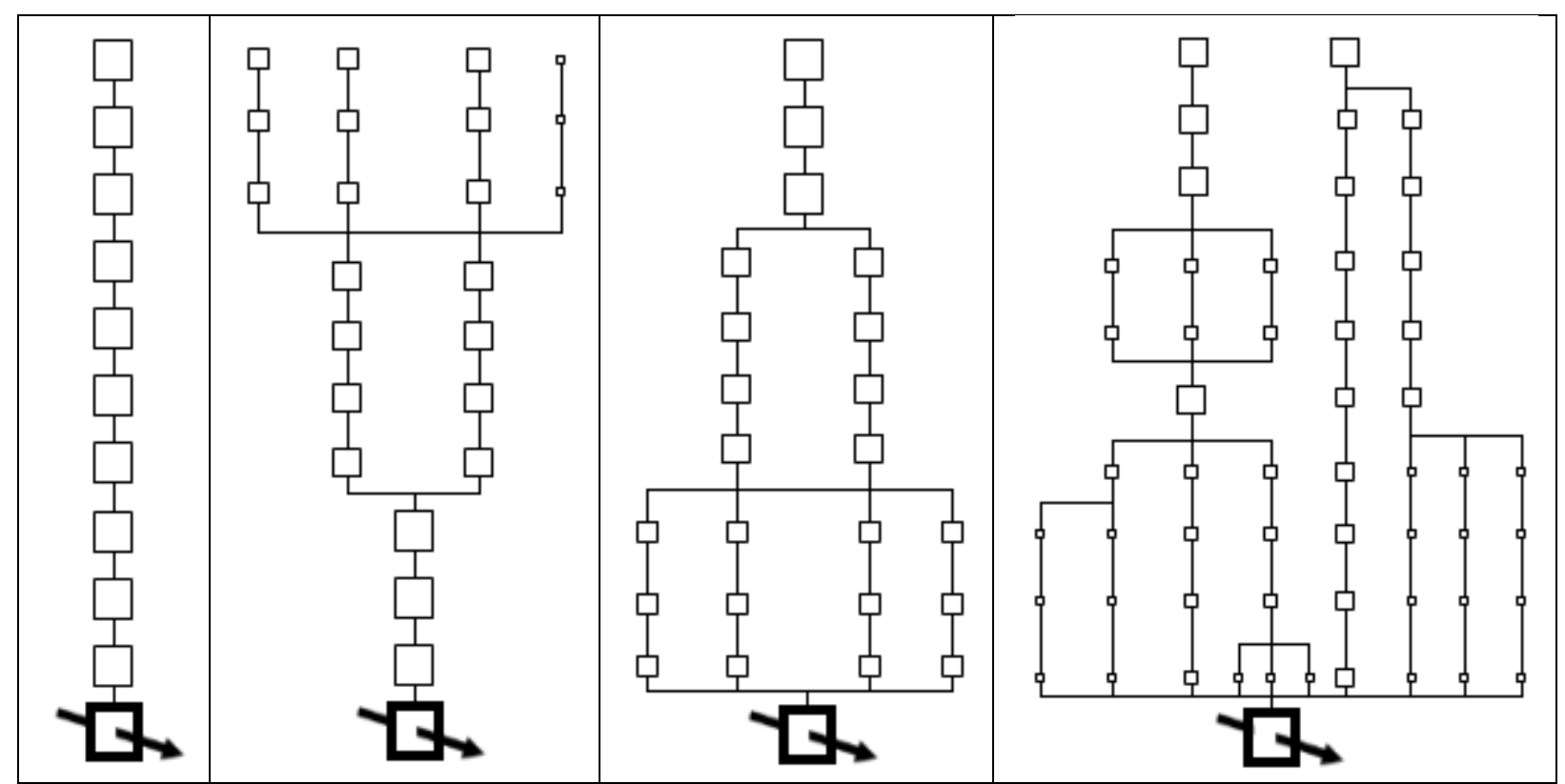

264 Figure 4: Diffusive porosity structures used to check the numerical implementation of the 265 SINC model, equivalent to the "MINC 1D" structure (left column). These structures display 266 the same behavior for homogeneous initial concentrations in the immobile zones. The mobile 267 zone is the bold box with the arrow. The size of the boxes is proportional to the porosity of 268 the compartments. Only the vertical distance of an immobile zone to the mobile zone is to 269 scale. 
270 For the structures displayed on Figure 2, the effective dispersion obtained with the MRMT

271 models converges quickly to the reference dispersion of the corresponding SINC model

272 (Figure 5). The equivalent MRMT model with only one immobile zone $(N=1)$, equivalent to

273 the double porosity model [Warren et al., 1963], already gives the right order of magnitude of

274 dispersion. With $N=2$, the error of the MRMT model is close to $10 \%$. With $N=4$, it is close to

$2751 \%$ and with $N=5$, it is close to $0.1 \%$. A very limited number of rates is thus sufficient to

276 represent even the complex diffusive structure displayed by Figure $2 \mathrm{~d}$. This fundamentally

277 comes from the homogenization nature of diffusion that systematically removes the extremes

278 of the concentration distributions as previously noted in numerous studies [Haggerty and

279 Gorelick, 1995; Villermaux, 1987].

280 In addition, the equivalent MRMT model with only one rate reproduces well the tailing of the

281 breakthrough curve (Figure 6). As expected, introducing higher rates progressively improves 282 the accuracy of the MRMT model at earlier times. The double peak observed for $N=1$ is a 283 classical feature of double porosity models where advection is much faster than diffusion in 284 the immobile porosity [Michalak and Kitanidis, 2000]. It vanishes for higher-order MRMT 285 models ( $N=2$ to 5 ), as higher rates enhance short-term mobile-immobile exchanges and 286 remove early breakthroughs.

287 The quality of the MRMT model with only very few rates fundamentally comes from the 288 dominating role of the smaller rates (i.e. larger transfer times). In fact the whole rate series as 289 determined by the algebraic diagonalization method shows that the lowest rate dominates in 290 every case by accounting for $70 \%$ to $85 \%$ of the total immobile porosity (Figure 7). The five 291 lowest rates represent at least $95 \%$ of the total immobile porosity for all the studied structures.

292 The evolution of the porosities $\phi_{i m}^{i}$ with the rates $\alpha_{i}$ is monotonic only for the MINC model 293 and becomes much more variable for more complex structures, highlighting the need of 294 identification methods that do not assume any a priori repartition of the immobile porosity 295 among the rates. We finally note that our results pertain to complex diffusive structures 296 observed on a given range of scales. If not close to the mobile zone, finer details would be fast 297 homogenized by diffusion and are unlikely to modify the identified rates. If close to the 298 mobile zone, finer details should be treated independently in the same way as for the larger299 scale structures and the MRMT models obtained at different scales should be eventually 300 superposed. 


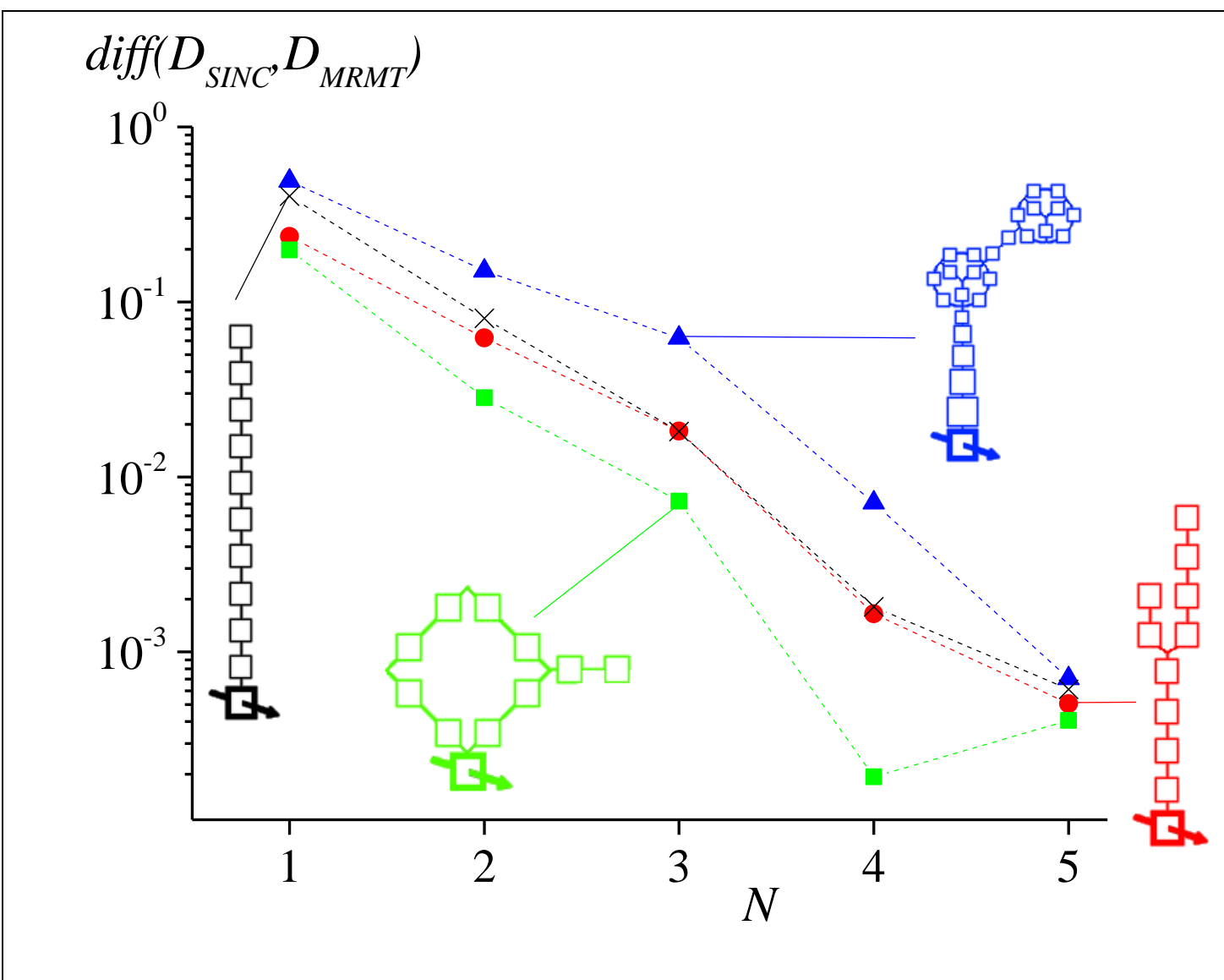

301 Figure 5: Differences in macrodispersions $\operatorname{diff}\left(D_{S I N C}, D_{M R M T}\right)$ (equation (20)) between SINC

302 models and their approximate MRMT models with a limited number of rates $N=1$ to5, for 303 the four SINC models presented in Figure 2. The determination of the approximate MRMT 304 models is achieved with the numerical identification method in the temporal domain (Section 3054.1 and Appendix C). 


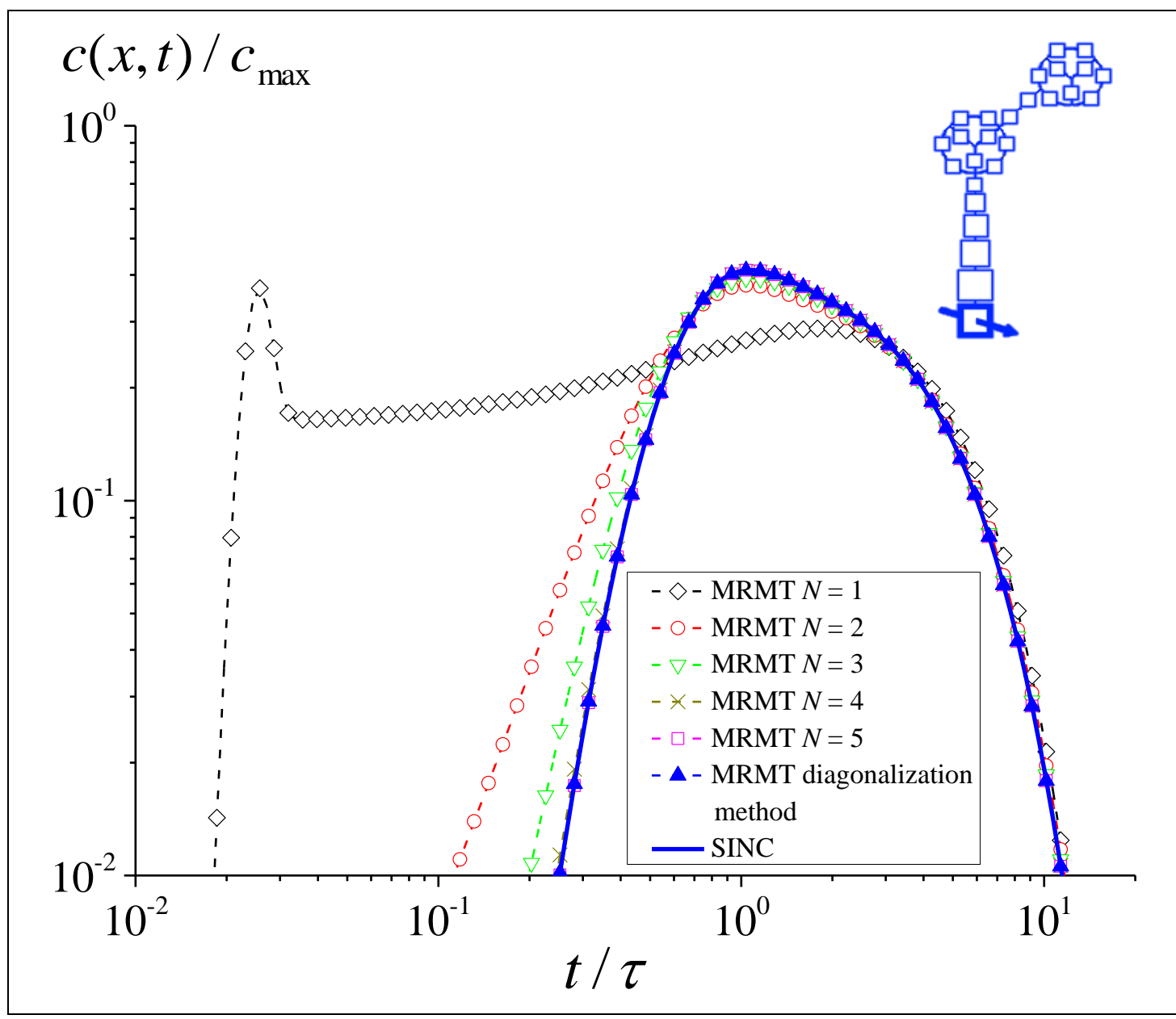

306 Figure 6: Breakthrough curves for the dissolution-like SINC model (Figure 2d) and for its 307 equivalent MRMT models, either determined by the diagonalization method (section 3), or by 308 the numerical method in the temporal domain with a limited number of $N$ rates (Section 4.1 309 and Appendix $C$ ). The concentrations are measured at the position $x=20 \sigma_{0}$. 


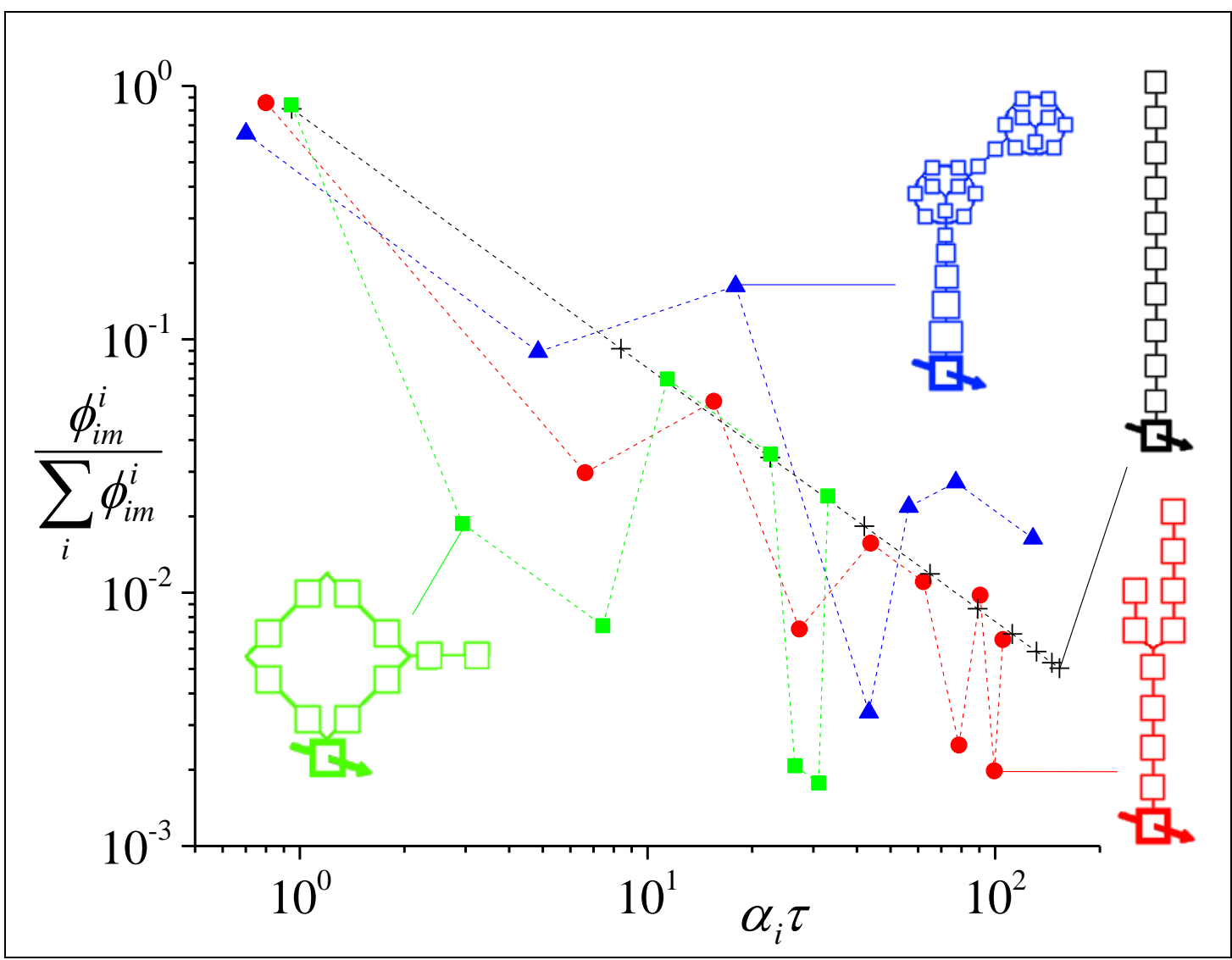

310 Figure 7: Normalized rates $\alpha_{i} \tau$ versus normalized porosities $\phi_{i m}^{i} / \sum_{i} \phi_{i m}^{i}$ for the MRMT

311 models equivalent to the four diffusive porosity structures presented in Figure 2, as 312 determined by the diagonalization method (section 3). Normalized rates larger than 200 or 313 corresponding to a normalized porosity smaller than $10^{-3}$ have been truncated. . 


\section{Conclusion}

315 We define a general mobile/immobile Structured INteracting Continua (SINC) transport 316 framework accounting for a broad variety of immobile porosity structures. Like in more 317 classical double porosity, Multi-Rate Mass Transfer (MRMT), and Multiple INteracting 318 Continua (MINC) frameworks, solute transport is dominated by advection in the mobile 319 porosity and is diffusion-like in the immobile porosity. The SINC framework introduces a connectivity pattern within the immobile zone covering a broad range of diffusive geological structures including cluster of dead-end fractures, irregular matrix shapes and dissolution patterns. Immobile structures are based on branching and looping structures, and on any combination of them. Solute transport is expressed as an advection-diffusion equation coupled to algebraically defined exchanges with a finite number of immobile zones. Interactions among the immobile zones and with the mobile zone are fully determined by a simple interaction matrix, which resumes to an arrow type of matrix in the MRMT case and to a tridiagonal matrix in the MINC case. The graph of the matrix registers the connectivity pattern while the value of its coefficients comes from relative porosities and strength of exchanges between immobile zones.

330 We show that any Structured INteracting Continua model is equivalent to a unique MRMT model, where the equivalence is defined as the strict identity of the concentrations in the mobile zone, whatever the initial and boundary conditions. The rates of the equivalent MRMT model are the eigenvalues of the subset of the interaction matrix where the line and column corresponding to the mobile zone are removed. The diagonalization method gives a first identification method of the equivalent MRMT with the same dimension, i.e. with the same number of immobile zones. Because of limitations coming essentially from the dimension of the immobile porosity structure, we set up alternative numerical methods designed to identify the most important rates controlling the transport of solute. Developed both in the temporal and Laplace domains, these methods seek for the combination of a finite number of exponential functions that best matches a simple discharge of the immobile zones within a quickly flushing mobile zone.

342 A simple sensitivity study on representative diffusive structures shows that very few rates are 343 needed for accurately modeling the solute transport in a 1D advection-dominated mobile zone 344 exchanging with an immobile porosity structure. Double porosity models (MRMT with a 345 single rate) already give the right order of magnitude of macrodispersion. Differences in 
346 macrodispersion drop down to around $10 \%$ for two rates, to $1 \%$ for four rates, and to less than $3470.1 \%$ for five rates. Simplified models based on only five rates approach accurately the 348 behavior of the system at intermediate to large times and only miss the very early responses.

349 While only few rates are necessary, their distribution and associated porosities are highly 350 variable, the complexity of the structure being transferred to the identified rates and 351 associated porosities. We thus conclude that MRMT models can be very efficient for 352 modeling diffusion-like transport in a broad range of porosity structures with only very few 353 rates. Even though numerical simulations have been done in 1D mobile domains, results are 354 likely generalize to 3D. Additional simulations should also be performed to investigate the 355 behavior of mixing and chemical reactivity both between different SINC structures and 356 between the SINC structures and their simplified MRMT counterparts. 


\section{Appendix A: Diagonalization of $\widehat{A}$}

We show that the eigenvalues of the matrix $\widehat{A}$ are real and negative and that they correspond to the opposites of the rates of the equivalent MRMT model $\left(-\alpha_{i}\right)$. As displayed by equations (4) and (7), $\widehat{A}=-\widehat{\Phi}^{-1} \widehat{M} \quad$ where $\quad \widehat{\Phi}=\Phi(2 \ldots N+1,2 \ldots N+1) \quad$ and $\widehat{M}=M(2 \ldots N+1,2 \ldots N+1) . \quad \widehat{\Phi}^{-1}$ is diagonal and its diagonal elements are all positive. Thus $\widehat{\Phi}^{-1}$ is positive definite, i.e. for every non-zero and real column vector $x$, $x^{T} \widehat{\Phi}^{-1} x=\sum_{i} \frac{x(i)^{2}}{\widehat{\Phi}(i, i)}>0 . \widehat{M}$ is symmetrical, real, diagonally dominant $|\widehat{M}(i, i)| \geq \sum_{j, j \neq i}|\hat{M}(i, j)|$, and strictly diagonally dominant $|\hat{M}(i, i)|>\sum_{j, j \neq i}|\hat{M}(i, j)|$ on its rows corresponding to the immobile zones connected to the mobile zone, due to the removal of the column corresponding to the mobile zone in the extraction of $\widehat{A}$ from $A$ (equation (7)). If $\widehat{A}$ is additionally of rank $N$, then $\widehat{A}$ is diagonalizable, has real eigenvalues, and has the same number of positive and negative eigenvalues as $-\widehat{M}$ [Horn, 1985].

We moreover show that $-\widehat{M}$ and equivalently $\widehat{A}$ have strictly negative, real eigenvalues. It is the direct consequence [Horn, 1985] of $-\widehat{M}$ being symmetrical, real, having only negative diagonal elements, and also being irreducible diagonally dominant. The diagonally dominance has been shown previously. The irreducibility property is more involved but can be proved by studying the properties of the graph defined by $\hat{M}$. When $\hat{M}$ represents an immobile structure connected to the mobile structure by a single link, at least one path exists from any immobile cell to any other immobile cell that does not cross the mobile zone, then the graph defined by $\hat{M}$ is strongly connected, so $\hat{M}$ is irreducible [Horn, 1985]. When several immobile zones are independently connected to the mobile zone, each of these immobile zones is associated to a strongly connected graph and to an irreducible diagonally dominant matrix, itself a sub-matrix of $\hat{M}$. The eigenvalues and eigenvectors of $\hat{M}$ are then obtained by clustering the ones of the sub-matrices. 


\section{Appendix B: Construction of the matrix $B$}

383 The norm of each eigenvector in $\hat{R}$ is defined up to a constant. A first straightforward step is 384 to adjust these norms so that the sum along the $2 . . N+1$ rows of $B$ is equal to zero. It is 385 achieved by taking $B_{i+1,1}=-\Lambda_{i}$, overly written in matrix form

$$
\left[\begin{array}{c}
B_{2,1} \\
\vdots \\
B_{N+1,1}
\end{array}\right]=-\Lambda\left[\begin{array}{c}
1 \\
\vdots \\
1
\end{array}\right]
$$

386 Given this choice and the properties of the matrices $A$ and $\hat{R}$, we demonstrate that the sum of 387 the elements of the first line of $B$ is also zero. We express the relation between the first 388 columns of $A$ and $B$ from equation (12):

$$
\left[\begin{array}{c}
B_{2,1} \\
\vdots \\
B_{N+1,1}
\end{array}\right]=\widehat{R}^{-1}\left[\begin{array}{c}
A_{2,1} \\
\vdots \\
A_{N+1,1}
\end{array}\right]
$$

389 As the sum of the elements of each line of $A$ is zero

$$
\left[\begin{array}{c}
A_{2,1} \\
\vdots \\
A_{N+1,1}
\end{array}\right]=-\widehat{A}\left[\begin{array}{c}
1 \\
\vdots \\
1
\end{array}\right]
$$

390 equation $(\mathrm{B} 2)$ rewrites

$$
\left[\begin{array}{c}
B_{2,1} \\
\vdots \\
B_{N+1,1}
\end{array}\right]=-\widehat{R}^{-1} \hat{A}\left[\begin{array}{c}
1 \\
\vdots \\
1
\end{array}\right]=-\Lambda \widehat{R}^{-1}\left[\begin{array}{c}
1 \\
\vdots \\
1
\end{array}\right] .
$$

391 By substituting equation (B4) into equation (B1), we deduce that the eigenvectors comply 392 with 


$$
\left[\begin{array}{l}
1 \\
\vdots \\
1
\end{array}\right]=R\left[\begin{array}{l}
1 \\
\vdots \\
1
\end{array}\right] .
$$

393 As $R^{-1} U$ corresponds to the concentrations in the equivalent model (equation (13)), equation 394 (B5) implies that a homogeneous immobile concentration profile in SINC remains unchanged 395 in the equivalent model. We finally express the sum of the $2 \ldots N+1$ elements of the first row of $396 \quad B$ and use the result of equation(B5):

$$
\begin{aligned}
& \sum_{j=2}^{N+1} B_{1, j}=\left[\begin{array}{lll}
B_{1,2} & \ldots & B_{1, N+1}
\end{array}\right]\left[\begin{array}{c}
1 \\
\vdots \\
1
\end{array}\right] \\
& =\left[\begin{array}{lll}
A_{1,2} & \ldots & A_{1, N+1}
\end{array}\right] \hat{R}\left[\begin{array}{c}
1 \\
\vdots \\
1
\end{array}\right] \\
& =\left[\begin{array}{lll}
A_{1,2} & \ldots & A_{1, N+1}
\end{array}\left[\begin{array}{c}
1 \\
\vdots \\
1
\end{array}\right]=\sum_{j=2}^{N+1} A_{1, j}=-A_{1,1}\right.
\end{aligned}
$$

397 An additional condition for $B$ to be representative of a MRMT model is $B_{1, j}>0$ for $398 j=2 \ldots N+1$. In the following we show that the adjustment of the norms of the eigenvectors is 399 sufficient to ensure this condition. Equations (12) and (B2) give: 


$$
\begin{aligned}
& {\left[\begin{array}{lll}
B_{1,2} & \ldots & B_{1, N+1}
\end{array}\right]} \\
& =\left[\begin{array}{lll}
A_{1,2} & \ldots & A_{1, N+1}
\end{array}\right] \widehat{R} \\
& =-\frac{1}{\phi_{m}}\left[\begin{array}{lll}
M_{1,2} & \ldots & M_{1, N+1}
\end{array}\right] \widehat{R} \\
& =-\frac{1}{\phi_{m}}\left[\begin{array}{lll}
M_{2,1} & \ldots & M_{N+1,1}
\end{array}\right] \widehat{R} \text { bec ause } M \text { is symmetric } \\
& =-\frac{1}{\phi_{m}}\left[\begin{array}{llll}
\phi_{i m}^{1} & A_{2,1} & \ldots & \phi_{i m}^{N} A_{N+1,1}
\end{array}\right] \widehat{R} \\
& =-\frac{1}{\phi_{m}}\left[\begin{array}{lll}
A_{2,1} & \ldots & A_{N+1,1}
\end{array}\right] \begin{array}{lll}
\phi_{i m}^{1} & \ddots \\
& \\
= & \frac{1}{\phi_{m}}\left[\begin{array}{llll}
B_{2,1} & \ldots & B_{N+1,1}
\end{array}\right] \widehat{R}^{T}\left[\begin{array}{lll}
\phi_{i m}^{1} & \\
& \ddots & \\
& & \phi_{i m}^{N}
\end{array}\right] \widehat{R}
\end{array} .
\end{aligned}
$$

400 We now show that the matrix $\widehat{R}^{T} \widehat{\Phi} \widehat{R}$ with $\widehat{\Phi}=\Phi(2 \ldots N+1,2 \ldots N+1)$ is diagonal with only 401 positive diagonal elements.

402 We note $\widehat{A}=-\widehat{\Phi}^{-1} \hat{M}$ with $\hat{M}=M(2 \ldots N+1,2 \ldots N+1) . \widehat{M}$ is symmetric, its diagonal 403 elements are positive, its non-zero off-diagonal elements are negative, but the sum of its 404 elements over each of its rows is not equal to zero. As the $\phi_{i m}^{i}$ are positive, we can consider 405 the root of the matrix $\widehat{\Phi}$ :

$$
\widehat{A}=\widehat{\Phi}^{-1 / 2} \underbrace{(-1) \widehat{\Phi}^{-1 / 2} \widehat{M} \widehat{\Phi}^{-1 / 2}}_{\widehat{\mathrm{C}}} \widehat{\Phi}^{1 / 2} .
$$

$406 \hat{C}$ is similar (in the mathematical sense) to $\hat{A}$, so it is diagonalizable and has the same 407 eigenvalues as $\hat{A}$. Moreover $\hat{C}$ is symmetric, so it can be diagonalized by an orthogonal 408 matrix $S$ :

$$
\widehat{C}=S \Lambda S^{-1}
$$

409 As a consequence, equation (B8) rewrites: 


$$
\begin{aligned}
\widehat{A} & =\widehat{\Phi}^{-1 / 2} S \Lambda S^{-1} \widehat{\Phi}^{1 / 2} \\
& =\widehat{\Phi}^{-1 / 2} S \Lambda\left(\widehat{\Phi}^{-1 / 2} S\right)^{-1}
\end{aligned} .
$$

410 As the norms of the eigenvectors in $\hat{R}$ are adjusted so the equation (B1) is verified, there 411 exists a unique orthogonal matrix $S$ such that

$$
S=\widehat{\Phi}^{1 / 2} \widehat{R}
$$

412 As $S$ is othogonal, $S^{T} S$ is diagonal with only positive diagonal elements and writes

$$
S^{T} S=\left(\widehat{\Phi}^{1 / 2} \widehat{R}\right)^{T} \widehat{\Phi}^{1 / 2} \widehat{R}=\widehat{R}^{T} \widehat{\Phi}^{1 / 2} \widehat{\Phi}^{1 / 2} \widehat{R}=\widehat{R}^{T} \widehat{\Phi} \hat{R}
$$

413 The matrix $\widehat{R}^{T} \widehat{\Phi} \widehat{R}$, which is present in equation (B7), is thus diagonal with only positive 414 diagonal elements. Consequently, as the $B_{1, j}$ are positive for $j=2 \ldots N+1$, so are the $B_{j, 1}$. 

model

418 We set up an optimization scheme to get MRMT models equivalent to a SINC model. We

419 first consider the case where the mass per unitary volume $m(t)$ discharged to an immobile 420 zone from a flushing experiment can effectively be modeled by a series of $N$ exponential 421 functions with rates $\alpha_{\mathrm{i}}$ and associate porosities $\phi_{i m}^{i}(m(t)=\gamma(t)$, see equation (15)). We 422 derive a set of equivalent expressions in the Laplace domain with simple dependences on $\alpha_{\mathrm{i}}$ 423 and $\phi_{i m}^{i}$. We then deduce optimization strategies both in the Laplace and temporal domains.

424 We assume first that the SINC model is strictly equivalent to a given MRMT model as in 425 section $2(m(t)=\gamma(t))$. It is the case when initial concentrations are homogeneous in the 426 immobile zone and when the immobile zones are constantly discharging to a quickly flushed 427 mobile zone where concentration is assumed to remain negligible ([Haggerty and Gorelick, 428 1995], Appendix B). In the Laplace domain, exponential functions become simple rational 429 functions and $\tilde{m}(p)=\tilde{\gamma}(p)$ is expressed as

$$
\tilde{m}(p)=\sum_{i=1}^{N} \frac{\phi_{i m}^{i} c_{0}}{\alpha_{i}} \frac{1}{1+\frac{p}{\alpha_{i}}}
$$

430 where $p$ is the Laplace variable and $\tilde{m}(p)$ (respectively $\tilde{\gamma}(p)$ ) is Laplace transform of $m(t)$ 431 (respectively $\gamma(p)$ ). We multiply equation $(\mathrm{C} 1)$ by the polynomial $P(p)$ of degree $N$

$$
P(p)=\prod_{i=1}^{N}\left(1+\frac{p}{\alpha_{i}}\right)=1+a_{1} p+a_{2} p^{2}+\ldots+a_{N} p^{N}
$$

432 and obtain

$$
\left(1+a_{1} p+a_{2} p^{2}+\ldots+a_{N} p^{N}\right) \tilde{m}(p)=\sum_{i=1}^{N}\left[\frac{\phi_{i m}^{i} c_{0}}{\alpha_{i}} \prod_{\substack{j=1 \\ j \neq i}}^{N}\left(1+\frac{p}{\alpha_{j}}\right)\right]
$$

433 If we now consider the polynomial $Q_{i}(p)$ of degree $N-1$ 


$$
Q_{i}(p)=\prod_{\substack{j=1 \\ j \neq i}}^{N}\left(1+\frac{p}{\alpha_{j}}\right)=1+a_{i, 1} p+a_{i, 2} p^{2}+\ldots+a_{i, N-1} p^{N-1}
$$

434 and substitute equation (C4) into equation (C3), we obtain:

$$
\left(a_{1} p+a_{2} p^{2}+\ldots+a_{N} p^{N}\right) \tilde{m}(p)+\tilde{m}(p)=\sum_{i=1}^{N} \frac{\phi_{i m}^{i} c_{0}}{\alpha_{i}}\left(1+a_{i, 1} p+a_{i, 2} p^{2}+\ldots+a_{i, N-1} p^{N-1}\right)
$$

435 The interest of equation (C5) is to be linear in the $a_{i}$ (polynomial coefficients of $P(p)$ ) and in $436 \phi_{i m}^{i} / \alpha_{i}$ with $i=1 \ldots N$. We isolate these quantities from the Laplace parameter-dependant 437 elements to obtain the linear system

$$
\varphi^{T}(p) \theta=y(p)
$$

438 with

$$
\varphi(p)=\left[\begin{array}{c}
p \tilde{m}(p) \\
p^{2} \tilde{m}(p) \\
\vdots \\
p^{N} \tilde{m}(p) \\
-1 \\
-p \\
\vdots \\
-p^{N-1}
\end{array}\right], \theta=\left[\begin{array}{c}
a_{1} \\
a_{2} \\
\vdots \\
a_{N} \\
\sum_{i=1}^{N} \frac{\phi_{i m}^{i} c_{0}}{\alpha_{i}} \\
\sum_{i=1}^{N} \frac{\phi_{i m}^{i} c_{0}}{\alpha_{i}} a_{i, 1} \\
\vdots \\
\sum_{i=1}^{N} \frac{\phi_{i m}^{i} c_{0}}{\alpha_{i}} a_{i, N-1}
\end{array}\right], y(p)=-\tilde{m}(p)
$$

439 Both $\varphi(p)$ and $\theta$ are vectors of dimension $2 N$. The rates $-\alpha_{i}$ are directly obtained from the 440 roots of the polynomial $P(p)$ of equation $(\mathrm{C} 2)$, whose coefficients are given by $\theta_{1} . . \theta_{N}$. The 441 porosities $\phi_{i m}^{i}$ are further deduced from $\theta_{N+1} . . \theta_{2 N}$ by inversing the $N+1 . .2 N$ equations of (C7):

$$
\left[\begin{array}{c}
\phi_{i m}^{1} \\
\phi_{i m}^{2} \\
\vdots \\
\phi_{i m}^{N}
\end{array}\right]=G^{-1}\left[\begin{array}{c}
\theta_{N+1} \\
\theta_{N+2} \\
\vdots \\
\theta_{2 N}
\end{array}\right]
$$

442 with 


$$
G=\left[\begin{array}{ccc}
c_{0} / \alpha_{1} & \cdots & c_{0} / \alpha_{N} \\
c_{0} a_{1,1} / \alpha_{1} & \cdots & c_{0} a_{N, 1} / \alpha_{N} \\
\vdots & & \vdots \\
c_{0} a_{1, N-1} / \alpha_{1} & \cdots & c_{0} a_{N, N-1} / \alpha_{N}
\end{array}\right]
$$

443 where the values of $a_{i, j}$ are deduced from the identified values of $\alpha_{i}$. In the case of strict 444 equivalence between MRMT and SINC models, the equivalent MRMT model can be found 445 through (C6)-(C9).

446 In the case where MRMT and SINC models are not strictly equivalent, we seek for the 447 composition of $N$ exponential functions that best matches $\tilde{m}(p)$ on a given sampling of the 448 Laplace parameter $p_{k}, k=1, \ldots, K$ of $p$ by using a least-square method, minimizing the 449 mismatch objective function $J$ [Garnier et al., 2008; Ljung, 1999]:

$$
J=\sum_{k=1}^{K}\left(y\left(p_{k}\right)-\varphi^{T}\left(p_{k}\right) \theta\right)^{2}
$$

450 The sampling should be extensive enough to contain all the information necessary to identify 451 the different rates. If $\alpha_{N}$ is the largest rate, the initial time sampling should be smaller than $452 \alpha_{N}^{-1} / 2$ following the spirit of Shannon's theorem. Adequate time sampling could then 453 increase with time for determining the smaller rates.

454 The minimum $\tilde{\theta}$ of $J$ is explicitly given by:

$$
\tilde{\theta}=\left(\sum_{k=1}^{K} \varphi\left(p_{k}\right) \varphi^{T}\left(p_{k}\right)\right)^{-1} \sum_{k=1}^{K} \varphi\left(p_{k}\right) y\left(p_{k}\right)
$$

455 and the $\alpha_{i}$ and $\phi_{i m}^{i}$ coefficients can be determined from the approximate $\tilde{\theta}_{i}$ coefficients and 456 (C6)-(C9).

$457 \tilde{\theta}$ can also be obtained in the temporal domain. We first divide equation (C5) by $p^{N}$ (which is 458 equivalent to integrate $N$ times over $t$ in the temporal domain) because of the better numerical 459 stability of integration compared to derivation 


$$
\left(\frac{1}{p^{N}}+a_{1} \frac{1}{p^{N-1}}+a_{2} \frac{1}{p^{N-2}}+\ldots+a_{N}\right) \tilde{m}(p)=\sum_{i=1}^{N} \frac{\phi_{i m}^{i} c_{0}}{\alpha_{i}}\left(\frac{1}{p^{N}}+a_{i, 1} \frac{1}{p^{N-1}}+a_{i, 2} \frac{1}{p^{N-2}}+\ldots+a_{i, N-1} \frac{1}{p}\right) .
$$

460 For convenience, we note

$$
\int^{(n)} m(u) d u=\int_{0}^{t} \int_{0}^{u_{1}} \int_{0}^{u_{2}} \ldots \int_{0}^{u_{n-1}} m\left(u_{n}\right) d u_{n} \ldots d u_{2} d u_{1} .
$$

461 The inverse Laplace transform of equation (C12) gives

$$
\begin{array}{r}
\int^{(N)} m(u) d u+a_{1} \int^{(N-1)} m(u) d u+a_{2} \int^{(N-2)} m(u) d u+\ldots+a_{N} m(t)= \\
\sum_{i=1}^{N} \frac{\phi_{i m}^{i} c_{0}}{\alpha_{i}}\left(\frac{t^{N-1}}{(N-1) !}+a_{i, 1} \frac{t^{N-2}}{(N-2) !}+a_{i, 2} \frac{t^{N-3}}{(N-3) !}+\ldots+a_{i, N-1}\right)
\end{array}
$$

462 As done previously in the Laplace domain, we separate the time-dependent elements from the 463 quantities depending on $\phi_{i m}^{i}$ and $\alpha_{i}$

$$
\varphi(t)=\left[\begin{array}{c}
\int^{(N-1)} m(u) d u \\
\int^{(N-2)} m(u) d u \\
\vdots \\
m(t) \\
-\frac{t^{N-1}}{(N-1) !} \\
-\frac{t^{N-2}}{(N-2) !} \\
\vdots \\
-1
\end{array}\right], \theta=\left[\begin{array}{c}
a_{1} \\
a_{2} \\
\vdots \\
a_{N} \\
\sum_{i=1}^{N} \frac{\phi_{i m}^{i} c_{0}}{\alpha_{i}} \\
\sum_{i=1}^{N} \frac{\phi_{i m}^{i} c_{0}}{\alpha_{i}} a_{i, 1} \\
\vdots \\
\sum_{i=1}^{N} \frac{\phi_{i m}^{i} c_{0}}{\alpha_{i}} a_{i, N-1}
\end{array}\right], y(t)=-\int^{(N)} m(u) d u
$$

$464 \theta$ may also be obtained with a similar least-square method by considering a discretization of 465 time $t_{k}(k=1 \ldots K)$ and by minimizing the objective function

$$
J=\sum_{k=1}^{K}\left(y\left(t_{k}\right)-\varphi^{T}\left(t_{k}\right) \theta\right)^{2}
$$

466 The minimum $\tilde{\theta}$ is given by

$$
\tilde{\theta}=\left(\sum_{k=1}^{K} \varphi\left(t_{k}\right) \varphi^{T}\left(t_{k}\right)\right)^{-1} \sum_{k=1}^{K} \varphi\left(t_{k}\right) y\left(t_{k}\right)
$$


$468 \quad N=2$

469 We recall the expression of the discharge of one immobile zone in MRMT model into a 470 mobile zone of constant concentration zero (equation (C1)):

$$
m(t)=\sum_{i=1}^{N} c_{0} \phi_{i m}^{i} \exp \left(-\alpha_{i} t\right)
$$

471 where $m(t)$ is the remaining mass per unitary volume of solute and $c_{0}$ is the initial 472 homogeneous immobile concentration.

473 Case $N=1$

474 In Laplace domain, equation (D1) rewrites:

$$
\begin{gathered}
\tilde{m}(p)=\frac{\phi_{i m}^{1} c_{0}}{\alpha_{1}} \frac{1}{1+\frac{p}{\alpha_{1}}} \\
\left(1+\frac{p}{\alpha_{1}}\right) \tilde{m}(p)=\frac{\phi_{i m}^{1} c_{0}}{\alpha_{1}}
\end{gathered}
$$

475 Dividing equation (D3) by $p$ and then using the inverse Laplace transform, we obtain:

$$
\int_{0}^{t} m(u) d u+\frac{m(t)}{\alpha_{1}}=\frac{\phi_{i m}^{1} c_{0}}{\alpha_{1}}
$$

476 Equations (D3) and (D4) are both linear in quantities depending on the unknown parameters $477 \quad \alpha_{1}$ and $\phi_{i m}^{1}$ to be identified. Equation (D4) can be written under the form:

$$
\varphi^{T}(t) \theta=y(t)
$$

478 with:

$$
\varphi(t)=\left[\begin{array}{c}
m(t) \\
-1
\end{array}\right], \theta=\left[\begin{array}{c}
1 / \alpha_{1} \\
c_{0} \phi_{i m}^{1} / \alpha_{1}
\end{array}\right], y(t)=-\int_{0}^{t} m(u) d u
$$

479 The vector $\tilde{\theta}$ which minimize the quantity: 


$$
J=\sum_{k=1}^{K}\left(y\left(t_{k}\right)-\varphi^{T}\left(t_{k}\right) \theta\right)^{2}
$$

480 with a discretization $t_{k}, k=1, \ldots, K$ of $t$, is given by:

$$
\tilde{\theta}=\left(\sum_{k=1}^{K} \varphi\left(t_{k}\right) \varphi^{T}\left(t_{k}\right)\right)^{-1} \sum_{k=1}^{K} \varphi\left(t_{k}\right) y\left(t_{k}\right)
$$

481 where

$$
\varphi\left(t_{k}\right) \varphi^{T}\left(t_{k}\right)=\left[\begin{array}{cc}
m\left(t_{k}\right)^{2} & -m\left(t_{k}\right) \\
-m\left(t_{k}\right) & 1
\end{array}\right], \quad \varphi\left(t_{k}\right) y\left(t_{k}\right)=\left[\begin{array}{c}
-m\left(t_{k}\right) \int_{0}^{t_{k}} m(u) d u \\
\int_{0}^{t_{k}} m(u) d u
\end{array}\right]
$$

482 so

$$
\tilde{\theta}=\left[\begin{array}{cc}
\sum_{k=1}^{K} m\left(t_{k}\right)^{2} & -\sum_{k=1}^{K} m\left(t_{k}\right) \\
-\sum_{k=1}^{K} m\left(t_{k}\right) & K
\end{array}\right]^{-1}\left[\begin{array}{c}
-\sum_{k=1}^{K} m\left(t_{k}\right) \int_{0}^{t_{k}} m(u) d u \\
\sum_{k=1}^{K} \int_{0}^{t_{k}} m(u) d u
\end{array}\right] .
$$

483 We then get $\alpha_{1}$ and $\phi_{i m}^{1}$ :

$$
\alpha_{1}=\frac{1}{\tilde{\theta}_{1}} \quad, \quad \phi_{i m}^{1}=\frac{\tilde{\theta}_{2} \alpha_{1}}{c_{0}}
$$

484 Case $N=2$

485 In Laplace domain, equation (D1) rewrites:

$$
\tilde{m}(p)=\frac{\phi_{i m}^{1} c_{0}}{\alpha_{1}} \frac{1}{1+\frac{p}{\alpha_{1}}}+\frac{\phi_{i m}^{2} c_{0}}{\alpha_{2}} \frac{1}{1+\frac{p}{\alpha_{2}}}
$$

486 which gives when multiplied by $\left(1+p / \alpha_{1}\right)\left(1+p / \alpha_{2}\right)$

$$
\left(1+p / \alpha_{1}\right)\left(1+p / \alpha_{2}\right) \tilde{m}(p)=\frac{\phi_{i m}^{1} c_{0}}{\alpha_{1}}\left(1+p / \alpha_{2}\right)+\frac{\phi_{i m}^{2} c_{0}}{\alpha_{2}}\left(1+p / \alpha_{1}\right)
$$


487 We divide then equation (D13) by $p^{2}$ (which is equivalent to integrate two times over $t$ )

$$
\frac{1}{\alpha_{1} \alpha_{2}} \tilde{m}(p)+\left(\frac{1}{\alpha_{1}}+\frac{1}{\alpha_{2}}\right) \frac{\tilde{m}(p)}{p}+\frac{\tilde{m}(p)}{p^{2}}=\frac{\phi_{i m}^{1} c_{0}}{\alpha_{1}}\left(\frac{1}{p^{2}}+\frac{1}{p \alpha_{2}}\right)+\frac{\phi_{i m}^{2} c_{0}}{\alpha_{2}}\left(\frac{1}{p^{2}}+\frac{1}{p \alpha_{1}}\right) .
$$

488 Then, by using the inverse Laplace transform, we obtain:

$$
\frac{1}{\alpha_{1} \alpha_{2}} m(t)+\left(\frac{1}{\alpha_{1}}+\frac{1}{\alpha_{2}}\right) \int_{0}^{t} m(u) d u+\int_{0}^{t} \int_{0}^{u} m(v) d v d u=\frac{\phi_{i m}^{1} c_{0}}{\alpha_{1}}\left(t+\frac{1}{\alpha_{2}}\right)+\frac{\phi_{i m}^{2} c_{0}}{\alpha_{2}}\left(t+\frac{1}{\alpha_{1}}\right) .
$$

489 Again equations (D12) and (D13) are both linear in quantities depending on the unknown 490 parameters $\alpha_{i}$ and $\phi_{i m}^{i}$ to be identified. Equation (D15) and can be written under the form:

$$
\varphi^{T}(t) \theta=y(t)
$$

491 with:

$$
\varphi(t)=\left[\begin{array}{c}
\int_{0}^{t} m(u) d u \\
m(t) \\
-t \\
-1
\end{array}\right], \theta=\left[\begin{array}{c}
1 / \alpha_{1}+1 / \alpha_{2} \\
1 /\left(\alpha_{1} \alpha_{2}\right) \\
\frac{\phi_{i m}^{1} c_{0}}{\alpha_{1}}+\frac{\phi_{i m}^{2} c_{0}}{\alpha_{2}} \\
\frac{\phi_{i m}^{1} c_{0}}{\alpha_{1} \alpha_{2}}+\frac{\phi_{i m}^{2} c_{0}}{\alpha_{1} \alpha_{2}}
\end{array}\right], y(t)=-\int_{0}^{t} \int_{0}^{u} m(v) d v d u
$$

492 The vector $\tilde{\theta}$ which minimizes the quantity:

$$
J=\sum_{k=1}^{K}\left(y(t)-\varphi^{T}(t) \theta\right)^{2}
$$

493 with a discretization $t_{k}, k=1, \ldots, K$ of $t$, is given by:

$$
\tilde{\theta}=\left(\sum_{k=1}^{K} \varphi\left(t_{k}\right) \varphi^{T}\left(t_{k}\right)\right)^{-1} \sum_{k=1}^{K} \varphi\left(t_{k}\right) y\left(t_{k}\right)
$$

494 We then have the following relations: 


$$
\left\{\begin{array}{cc}
1 / \alpha_{1}+1 / \alpha_{2} & =\tilde{\theta}_{1} \\
1 /\left(\alpha_{1} \alpha_{2}\right) & =\tilde{\theta}_{2} \\
\frac{\phi_{i m}^{1} c_{0}}{\alpha_{1}}+\frac{\phi_{i m}^{2} c_{0}}{\alpha_{2}} & =\tilde{\theta}_{3} \\
\frac{\phi_{i m}^{1} c_{0}}{\alpha_{1} \alpha_{2}}+\frac{\phi_{i m}^{2} c_{0}}{\alpha_{1} \alpha_{2}}=\tilde{\theta}_{4}
\end{array}\right.
$$

495 from which we can identify the unknown parameters $-\alpha_{1}$ and $-\alpha_{2}$ may be obtained as the 496 roots of the polynomial $1+\tilde{\theta}_{1} x+\tilde{\theta}_{2} x^{2}$. Once $\alpha_{1}$ and $\alpha_{2}$ are identified, $\phi_{i m}^{1}$ and $\phi_{i m}^{2}$ are given 497 by:

$$
\left[\begin{array}{l}
\phi_{i m}^{1} \\
\phi_{i m}^{2}
\end{array}\right]=\frac{1}{c_{0}}\left[\begin{array}{cc}
1 / \alpha_{1} & 1 / \alpha_{2} \\
1 / \alpha_{1} \alpha_{2} & 1 / \alpha_{1} \alpha_{2}
\end{array}\right]^{-1}\left[\begin{array}{c}
\tilde{\theta}_{3} \\
\tilde{\theta}_{4}
\end{array}\right] .
$$




\section{Acknowledgements}

The ANR is acknowledged for its funding through its project $\mathrm{H} 2 \mathrm{MNO} 4$ under the number ANR-12-MONU-0012-01. The authors are also grateful to Linda Luquot, Alain Rapaport and Jesús Carrera for stimulating discussions, and additionally to Linda Luquot for providing the illustrative dissolution patterns. We also thank the four anonymous reviewers for their insightful and critical review of the manuscript, as well as Cass Miller for his editorial work. 


\section{References}

Benson, D. A., and M. M. Meerschaert (2009), A simple and efficient random walk solution of multirate mobile/immobile mass transport equations, Advances in Water Resources, 32(4), 532-539, doi:http://dx.doi.org/10.1016/j.advwatres.2009.01.002.

Benson, D. A., R. Schumer, M. M. Meerschaert, and S. W. Wheatcraft (2001), Fractional dispersion, Lévy motion, and the MADE tracer test, Transport in Porous Media, 42(1-2), 211-240, doi:10.1007/978-94-017-1278-1_11.

Benson, D. A., S. W. Wheatcraft, and M. M. Meerschaert (2000), Application of a fractional advection-dispersion equation, Water Resources Research, 36(6), doi:http://dx.doi.org/10.1029/2000WR900031.

Berkowitz, B., A. Cortis, M. Dentz, and H. Scher (2006), Modeling non-Fickian transport in geological formations as a continuous time random walk, Reviews of Geophysics, 44(2), doi:10.1029/2005rg000178.

Berkowitz, B., and H. Scher (1998), Theory of anomalous chemical transport in random fracture networks, Physical Review E, 57(5), doi:http://dx.doi.org/10.1103/PhysRevE.57.5858.

Berkowitz, B., H. Scher, and S. Silliman (2000), Anomalous transport in laboratory-scale, heterogeneous porous media, Water Resources Research, 36(1), doi:http://dx.doi.org/10.1029/1999WR900295.

Carrera, J., X. Sánchez-Vila, I. Benet, A. Medina, G. Galarza, and J. Guimerà (1998), On matrix diffusion: formulations, solution methods and qualitative effects, Hydrogeology Journal, 6(1), doi:http://dx.doi.org/10.1007/s100400050143.

Cushman, J. H., and T. R. Ginn (2000), Fractional advection-dispersion equation: A classical mass balance with convolution-Fickian flux, Water Resources Research, 36(12), doi:http://dx.doi.org/10.1029/2000WR900261.

de Dreuzy, J. R., A. Rapaport, T. Babey, and J. Harmand (2013), Influence of porosity structures on mixing-induced reactivity at chemical equilibrium in mobile/immobile Multi-Rate Mass Transfer (MRMT) and Multiple INteracting Continua (MINC) models, Water Resources Research, 49(12), 85118530, doi:10.1002/2013WR013808.

Dentz, M., and B. Berkowitz (2003), Transport behavior of a passive solute in continuous time random walks and multirate mass transfer, Water Resources Research, 39(5), 1111, doi:10.1029/2001WR001163.

Dentz, M., A. Cortis, H. Scher, and B. Berkowitz (2004), Time behavior of solute transport in heterogeneous media: transition from anomalous to normal transport, Advances in Water Resources, 27(2), 155-173, doi:http://dx.doi.org/10.1016/j.advwatres.2003.11.002.

Fernandez-Garcia, D., G. Llerar-Meza, and J. J. Gomez-Hernandez (2009), Upscaling transport with mass transfer models: Mean behavior and propagation of uncertainty, Water Resources Research, 45, doi:10.1029/2009wr007764.

Flekkøy, E. G., A. Malthe-Sørenssen, and B. Jamtveit (2002), Modeling hydrofracture, J. Geophys. Res., 107(B8), 2151.

Garnier, H., L. Wang, and P. Young (2008), Direct Identification of Continuous-time Models from Sampled Data: Issues, Basic Solutions and Relevance, in Identification of Continuous-time Models from Sampled Data, edited by H. Garnier and L. Wang, pp. 1-29, Springer London, doi:10.1007/978-184800-161-9_1. 
Golfier, F., M. Quintard, F. Cherblanc, B. A. Zinn, and B. D. Wood (2007), Comparison of theory and experiment for solute transport in highly heterogeneous porous medium, Advances in Water Resources, 30(11), 2235-2261, doi:10.1016/j.advwatres.2007.05.004.

Golfier, F., C. Zarcone, B. Bazin, R. Lenormand, D. Lasseux, and M. Quintard (2002), On the ability of a Darcy-scale model to capture wormhole formation during the dissolution of a porous medium, Journal of Fluid Mechanics, 457, 213-254, doi:10.1017/s0022112002007735.

Gotovac, H., V. Cvetkovic, and R. Andricevic (2009), Flow and travel time statistics in highly heterogeneous porous media, Water Resources Research, 45, 24, doi:http://dx.doi.org/10.1029/2008WR007168.

Gouze, P., Y. Melean, T. Le Borgne, M. Dentz, and J. Carrera (2008), Non-Fickian dispersion in porous media explained by heterogeneous microscale matrix diffusion, Water Resources Research, 44(11), 19, doi:http://dx.doi.org/10.1029/2007wr006690.

Haggerty, R. (2001), Matrix diffusion - heavy-tailed residence time distributions and their influence on radionuclide retention, paper presented at 5th GEOTRAP Workshop on Radionuclide Retention in Geologic Media,May 7-9, 2001, Organisation for Economic Co-Operation and Development, Nuclear Energy Agency, 75 - Paris (France), Oskarshamn, Sweden, 2002.

Haggerty, R., S. W. Fleming, L. C. Meigs, and S. A. McKenna (2001), Tracer tests in a fractured dolomite 2. Analysis of mass transfer in single-well injection-withdrawal tests, Water Resources Research, 37(5), doi:http://dx.doi.org/10.1029/2000WR900334.

Haggerty, R., and S. M. Gorelick (1995), Multiple-Rate Mass Transfer for Modeling Diffusion and Surface Reactions in Media with Pore-Scale Heterogeneity, Water Resources Research, 31(10), 23832400, doi:10.1029/95WR10583.

Haggerty, R., C. F. Harvey, C. F. von Schwerin, and L. C. Meigs (2004), What controls the apparent timescale of solute mass transfer in aquifers and soils? A comparison of experimental results, Water Resources Research, 40(1), doi:10.1029/2002wr001716.

Haggerty, R., S. A. McKenna, and L. C. Meigs (2000), On the late-time behavior of tracer test breakthrough curves, Water Resources Research, 36(12), 3467-3479, doi:http://dx.doi.org/10.1029/2000WR900214.

Horn, R. A. (1985), Matrix analysis / Roger A. Horn, Charles R. Johnson, Cambridge University Press, Cambridge; New York.

Jardine, P. M., W. E. Sanford, J. P. Gwo, O. C. Reedy, D. S. Hicks, J. S. Riggs, and W. B. Bailey (1999), Quantifying diffusive mass transfer in fractured shale bedrock, Water Resources Research, 35(7), 2015-2030, doi:10.1029/1999WR900043.

Karimi-Fard, M., B. Gong, and L. J. Durlofsky (2006), Generation of coarse-scale continuum flow models from detailed fracture characterizations, Water Resources Research, 42(10), doi:http://dx.doi.org/10.1029/2006WR005015.

Ljung, L. (1999), System identification : theory for the user, Prentice Hall, Upper Saddle River.

Luquot, L., O. Rodriguez, and P. Gouze (2014), Experimental Characterization of Porosity Structure and Transport Property Changes in Limestone Undergoing Different Dissolution Regimes, Transp Porous Med, 101(3), 507-532, doi:10.1007/s11242-013-0257-4.

Michalak, A. M., and P. K. Kitanidis (2000), Macroscopic behavior and random-walk particle tracking of kinetically sorbing solutes, Water Resources Research, 36(8), 2133-2146, doi:10.1029/2000wr900109. 
Neretnieks, I. (1980), Diffusion in the Rock Matrix: An Important Factor in Radionucleside Retardation?, Journal of Geophysical Research, 85(B8), 4379-4397, doi:http://dx.doi.org/10.1029/JB085iB08p04379.

Neuman, S. P., and D. M. Tartakovsky (2009), Perspective on theories of non-Fickian transport in heterogeneous media, Advances in Water Resources, 32(5), 670-680, doi:10.1016/j.advwatres.2008.08.005.

Noetinger, B., and T. Estebenet (2000), Up-scaling of double porosity fractured media using continuous-time random walks methods, Transport in Porous Media, 39(3).

Pruess, K. (1992), Brief Guide to the MINC - Method for Modeling Flow and Transport in Fractured MediaRep., Earth Sciences Division, Lawrence Berkeley National Laboratory. Berkeley CA USA.

Pruess, K., and T. N. Narasimhan (1985), A practical method for modeling fluid and heat-flow in fractured porous-media, Society of Petroleum Engineers Journal, 25(1), 14-26, doi:http://dx.doi.org/10.2118/10509-PA.

Silva, O., J. Carrera, M. Dentz, S. Kumar, A. Alcolea, and M. Willmann (2009), A general real-time formulation for multi-rate mass transfer problems, Hydrology and Earth System Sciences, 13(8), 1399-1411, doi:http://dx.doi.org/10.5194/hess-13-1399-2009.

Sornette, A., P. Davy, and D. Sornette (1993), Fault Growth in Brittle-Ductile Experiments and the Mechanics of Continental Collisions, Journal of Geophysical Research-Solid Earth, 98(B7), 1211112139, doi:http://dx.doi.org/10.1029/92JB01740.

Sudicky, E. A., and E. O. Frind (1982), Contaminant transport in fractured porous-media - analytical solutions for a system of parallel fractures, Water Resources Research, 18(6), 1634-1642, doi:http://dx.doi.org/10.1029/WR018i006p01634.

Tang, D. H., E. O. Frind, and E. A. Sudicky (1981), Contaminant transport in fractured porous-media analytical solution for a single fracture, Water Resources Research, 17(3), 555-564, doi:http://dx.doi.org/10.1029/WR017i003p00555.

Tsang, Y. W. (1995), Study of alternative tracer tests in characterizing transport in fractured rocks, Geophysical Research Letters, 22(11), 1421-1424, doi:10.1029/95GL01093.

Villermaux, J. (1987), Chemical-engineering approach to dynamic modeling of linear chromatography - a flexible method for representing complex phenomena from simple concepts, Journal of Chromatography, 406, 11-26, doi:10.1016/s0021-9673(00)94014-7.

Warren, J. E., P. J. Root, and M. Aime (1963), The Behavior of Naturally Fractured Reservoirs, Society of Petroleum Engineers Journal, September, 245-255, doi:http://dx.doi.org/10.2118/426-PA.

Willmann, M., J. Carrera, and X. Sanchez-Vila (2008), Transport upscaling in heterogeneous aquifers: What physical parameters control memory functions?, Water Resources Research, 44(12), doi:10.1029/2007wr006531. 


\section{Figure captions}

Figure 1: (a) Skeleton of a dissolution feature in an oolitic limestone, observed by X-ray micro-tomography [Luquot et al., 2014]. The dissolving acidic solution percolates from top to bottom on the general view (bottom left). Its $\mathrm{pH}$ increases from top to bottom and from inside out of the main flow path indicated by the curved arrow on the detailed view (top right). The acid dissolves preferentially the calcite cement surrounding the oolites, the size of the pores progressively decreases away from the main flow path, and the organization of the pores becomes more complex. (b) Structured INteracting Continua model (SINC) sketched from the dissolution pattern of (a) with three cross sections transversal to the mobile zone materialized by the arrow. (c) Equivalent MRMT model with the 5 most important rates as determined by the numerical methods set up in section 4. The size of the boxes scales with the porosity affected to the rates labeled by triangles in Figure 7.

Figure 2: Examples of Structured INteracting Continua (SINC) used to illustrate and validate the numerical identification methods of the equivalent MRMT models. From left to right, the diffusive porosity structures are (a) the classical Multiple INteracting Continua (MINC) [Pruess and Narasimhan, 1985], (b) an asymmetric $\mathrm{Y}$ with a single junction, (c) an asymmetric loop, and (d) the dissolution structure presented in Figure 1. The size of the immobile cells is proportional to their porosity and the distance along the immobile structure is to scale. The mobile zone is represented by the thick black box with the crossing arrow. Its size has been exaggerated 10 times to be clearly marked. To be comparable, the four structures have the same total porous volume and the same radius of gyration taken with respect to the mobile zone.

Figure 3: Diffusive porosity structures represented as cross-sections transversal to the mobile zone direction ((a),(b)), with their associated interaction matrix $A((\mathrm{c}),(\mathrm{d}))$ for the asymmetric Y (top) and MRMT structures (bottom). Dotted frames around subsets of the immobile porosity structures ((a) and (b)) and around matrix lines ((c) and (d)) show how structures are translated in matrix form. Parameters for the asymmetric Y structure are taken from Table 1 and the multiplicative factor $\eta(\eta=5.015)$ is equal to the ratio of the distance between two consecutive immobile zones to the radius of gyration of the immobile domain to the mobile zone. $\beta$ is the ratio of the total immobile porosity to the mobile porosity. 
Figure 4: Diffusive porosity structures used to check the numerical implementation of the SINC model, equivalent to the "MINC 1D" structure (left column). These structures display the same behavior for homogeneous initial concentrations in the immobile zones. The mobile zone is the bold box with the arrow. The size of the boxes is proportional to the porosity of the compartments. Only the vertical distance of an immobile zone to the mobile zone is to scale.

Figure 5: Differences in macrodispersions $\operatorname{diff}\left(D_{\text {SINC }}, D_{M R M T}\right)$ (equation (20)) between SINC models and their approximate MRMT models with a limited number of rates $N=1$ to5, for the four SINC models presented in Figure 2. The determination of the approximate MRMT models is achieved with the numerical identification method in the temporal domain (Section 4.1 and Appendix $C$ ).

Figure 6: Breakthrough curves for the dissolution-like SINC model (Figure 2d) and for its equivalent MRMT models, either determined by the diagonalization method (section 3), or by the numerical method in the temporal domain with a limited number of $N$ rates (Section 4.1 and Appendix $C$ ). The concentrations are measured at the position $x=20 \sigma_{0}$.

Figure 7: Normalized rates $\alpha_{i} \tau$ versus normalized porosities $\phi_{i m}^{i} / \sum_{i} \phi_{i m}^{i}$ for the MRMT models equivalent to the four diffusive porosity structures presented in Figure 2, as determined by the diagonalization method (section 3). Normalized rates larger than 200 or corresponding to a normalized porosity smaller than $10^{-3}$ have been truncated. . 